HU-EP-02/59

hep-th/0212233

\title{
Superpotential of the $M$-theory conifold and type IIA string theory
}

\author{
Gottfried Curio \\ Humboldt Universität zu Berlin, Institut für Physik, \\ Invalidenstrasse 110, 10115 Berlin, Germany
}

\begin{abstract}
The membrane instanton superpotential for $M$-theory on the $G_{2}$ holonomy manifold given by the cone on $\mathbf{S}^{\mathbf{3}} \times \mathbf{S}^{\mathbf{3}}$ is given by the dilogarithm and has Heisenberg monodromy group in the quantum moduli space. We compare this to a Heisenberg group action on the type IIA hypermultiplet moduli space for the universal hypermultiplet, to metric corrections from membrane instantons related to a twisted dilogarithm for the deformed conifold and to a flat bundle related to a conifold period, the Heisenberg group and the dilogarithm appearing in five-dimensional Seiberg/Witten theory.
\end{abstract}




\section{Introduction}

The goal of this paper, which may be read as a companion paper to [8], is to put into perspective the occurrence of the dilogarithm and the Heisenberg group action for the $M$-theory conifold via comparison to analogous phenomena in type IIA string theory (which are not just treating the identical problem of describing the superpotential [7]).

In the conifold transition between Calabi-Yau manifolds in type II string theory an $\mathbf{S}^{\mathbf{3}}$ is exchanged with an $\mathbf{S}^{\mathbf{2}}$. When lifted to $M$-theory [1], [6] the resulting geometries become symmetrical: the two small resolutions on the one side of the transition become $\mathbf{S}^{\mathbf{3}}$ 's as well, Hopf fibred by the $M$-theory circle $\mathbf{S}_{\mathbf{1 1}}^{\mathbf{1}}$. This corresponds in type IIA to having one unit of RR flux on the $\mathbf{S}^{2}$; on the deformed conifold side one has a D6 brane wrapped on the $\mathbf{S}^{\mathbf{3}}$. $M$-theory of the corresponding non-compact $G_{2}$ holonomy manifold (with covariant constant three-form $\Upsilon$ ) is a deformation $X_{7}$ of the cone over $\mathbf{S}^{\mathbf{3}} \times \mathbf{S}^{\mathbf{3}}=S U(2)^{3} / S U(2)_{\text {diag }}$. It is topologically $\mathbf{R}^{\mathbf{4}} \times \mathbf{S}_{\mathbf{Q}}^{\mathbf{3}}$ (where $\mathbf{R}^{\mathbf{4}}$ comes from the 'filled in' $\mathbf{S}_{\mathbf{D}}^{\mathbf{3}}$ ) and comes in three triality equivalent versions $X_{1}, X_{2}, X_{3}$ [1], [7], [8].

The superpotential given by the multi-cover membrane instantons is given by the dilogarithm [7], [8] of the modulus $u=e^{i \Phi}$ where $\Phi=\int_{Q} C+i \Upsilon$

$$
L i(u)=\sum_{n=1} \frac{u^{n}}{n^{2}}=-\int_{0}^{u} \log (1-u) d \log u
$$

The multi-valuedness is described by the monodromy representation of the fundamental group $\pi_{1}\left(\mathbf{P}^{\mathbf{1}} \backslash\{0,1, \infty\}\right)$. The relevant local system is given by a bundle, flat with respect to a suitable connection. In the case of the logarithm the monodromy of $\log z$ around $z=0$ is described by adding integers (times $2 \pi i$ ) what is captured by the monodromy

matrix $\left(\begin{array}{cc}1 & 2 \pi i \\ 0 & 1\end{array}\right)$ acting on the two-vector $(\log z, 1)^{t}$; the generalisation in the case of the dilogarithm involves the Heisenberg group of upper triangular $3 \times 3$ matrices acting on the three-vector $c_{3}$. The Heisenberg bundle gives for the monodromy at infinity [8]

$$
c_{3}=\left(\begin{array}{c}
L i(y) \\
\log y \\
1
\end{array}\right) \quad, \quad M\left(l_{\infty}\right)=\left(\begin{array}{ccc}
1 & 2 \pi i & 0 \\
0 & 1 & -2 \pi i \\
0 & 0 & 1
\end{array}\right)
$$

Now let us compare to the type IIA results. In the case of the (deformed) conifold one has a relation between the quaternionic type IIA modulus and the $M$-theory modulus. The metric on the hypermultiplet moduli space is corrected by membrane instantons [2] wrapping the $\mathbf{S}^{\mathbf{3}}$ and, taking into account normalizations [13], (a twisted version of) $L i$ of the corresponding $\mathbf{S}^{\mathbf{3}}$ modulus occurs in a suitable hyperkähler limit of the quaternionic 
moduli space geometry (where the details of the embedding of the local conifold $T^{*} \mathbf{S}^{\mathbf{3}}$ in a specific global Calabi-Yau become irrelevant). Here the $N=1$ superpotential $W=\operatorname{Li}(u)$ corresponds to the $N=2$ metric corrections just as one and the same Schwinger computation has different physical interpretations in $N=0,1,2$ (cf. below).

Going beyond that limit but keeping the aimed at universality we further compare with the moduli space of the universal hypermultiplet (cf. [14] - [17]) and a Heisenberg action on it. In the $M$-theory conifold the quantities $\int_{\mathbf{S}_{\mathbf{Q} / \mathbf{D}}^{3}} *_{7} G$ (analogous to certain type IIA membrane charges, cf. sect. 2) accompany in the scalar potential term $\int_{D} C \cdot \int_{Q} *_{7} G$ (the imaginary parts of) $\log \eta=\log \beta u$ and $\log u$ which occupy places in the Heisenberg geometry (2.3) corresponding to the positions in the Heisenberg geometry (2.14) of the type IIA membrane charge parameters $\beta, \gamma$.

Our second theme is to compare to five-dimensional Seiberg/Witten theory on $\mathbf{R}^{4} \times$ $\mathbf{S}_{\mathbf{R}}^{1}$ ([26] - [29]). There the dilogarithm $L i$ occurs when describing the dual period $A_{D}$ in the flat bundle of $5 \mathrm{D} N=2 S U(2)$ field theory. Note that the $4 \mathrm{D}$ period $a_{D}$ is related to the conifold ${ }^{1}$. In the weak coupling limit one has for the monodromy at infinity (in suitably normalized periods and measuring the quantum scale $\Lambda$ by the compactification scale $m_{R}=1 / R$ in terms of the dimensionless quantity $\zeta=\frac{\Lambda}{m_{R}}$ )

$$
\left(\begin{array}{c}
\tilde{\mathcal{A}_{D}} \\
\tilde{\mathcal{A}} \\
1
\end{array}\right)=\left(\begin{array}{ccc}
1 & \log \zeta & 0 \\
0 & 1 & 0 \\
0 & 0 & 1
\end{array}\right)\left(\begin{array}{c}
L i(x) \\
\log x \\
1
\end{array}\right) \quad, \quad \tilde{M}_{\infty}=\left(\begin{array}{ccc}
1 & -2 \cdot 2 \pi i & 2 \pi i \log \zeta \\
0 & 1 & 2 \cdot 2 \pi i \\
0 & 0 & 1
\end{array}\right)
$$

Therefore one has that the $5 \mathrm{D}$ analogue $\tilde{\mathcal{A}_{D}}$ of the conifold period $a_{D}$ is given by the dilogarithm of (the exp of) $\tilde{\mathcal{A}}$ up to a linear modification (cf. [8], sect. 4.4)

$$
\tilde{\mathcal{A}}_{D}=\operatorname{Li}\left(e^{\tilde{\mathcal{A}}}\right)+(\log \zeta) \tilde{\mathcal{A}}
$$

We will also remark on relations between the monodromy at the conifold point (in a type IIB vector multiplet moduli space language) and $M$-theory results, and on relations between the way of derivation of the membrane instanton superpotential (1.1) and an instanton reinterpretation [12] of the occurrence of $L i$ as an imaginary energy density $L i\left(e^{2 \pi i \tau}\right.$ ) (with $\tau=i \frac{m^{2}}{2 e E}$, giving the probability density for the pair creation in a constant electric background $E$ ) in the classical non-supersymmetric Schwinger computation.

Note the different meanings of $L i$ : the pair production rate $w$ (related to the vacuum transition amplitude $P_{0}$ ) for $N=0$ (Schwinger), the disc instanton superpotential

\footnotetext{
${ }^{1}$ at the monopole point $\tilde{u}=1$, i.e. $\Xi_{6} \sim a_{D} \sim x_{+} \sim \tilde{u}-1$, by (D.15), (E.9), where $\Xi_{6}$ is the period related to the 6-cycle in type IIA respectively the vanishing $\mathbf{S}^{\mathbf{3}}$ of the conifold in type IIB
} 
$W=F_{0,1}$ (an open topological string amplitude) respectively the membrane instanton superpotential for $N=1$, the $R^{2}$-correction $F_{1}$ (a closed topological string amplitude) for $N=2$ (cf. app. F and footn. 10), and the metric correction (2.28).

This paper is organized as follows. In Section 2 we compare the $M$-theory situation with the type IIA hypermultiplet moduli space: we consider a Heisenberg action on it for the universal hypermultiplet and point to a relation of a twisted dilogarithm with metric corrections for the deformed conifold, point to $M$-theory relations reflecting the conifold monodromy and parallelisms of the $M$-theory treatment of the membrane instanton superpotential with an instanton description of the classical $(N=0)$ Schwinger computation. In Section 3 we describe how $L i$ occurs, related to the 5D analogue of the conifold period $a_{D}$, in the three-dimensional flat bundle of $N=2$ pure $S U(2)$ gauge theory in 5D. In Section 4 we collect the observations in an interpretational discussion. In the appendix we recall background for the 5D Seiberg/Witten theory, as well as on stringy resp. $M$-theoretic embeddings, and on the Schwinger computation.

\section{Structures in the type IIA hypermultiplet moduli space}

A hypermultiplet of the effective four-dimensional theory of the type IIA string on a Calabi-Yau has a quaternionic modulus consisting of two complex parts. One is given by the integral of the holomorphic three-form $\Omega$ over a three-cycle $A=\mathbf{S}^{\mathbf{3}}$, say, the other by the integral of the Ramond three-form $C$ over $A$ and a dual cycle $B$. In aiming at a certain universality different approaches are possible. Either one can localise and consider the deformed conifold $T^{*} \mathbf{S}^{\mathbf{3}}$; this concerns a hypermultiplet related to a specific three-cycle in the Calabi-Yau manifold $X$, but zooming to the local limit (hyperkähler reduction of the quaternionic geometry) gives those results a certain universality (cf. remark 1 below). This makes them amenable to a comparison with the other possible approach which treats the universal hypermultiplet.

The conifold transition in type IIA and $M$-theory

In a type IIA reinterpretation (cf. [1]) one divides by the circle $\mathbf{S}_{\mathbf{1 1}}^{\mathbf{1}}=U(1) \subset S U(2)_{1}$ giving for $X_{1}=\mathbf{R}^{\mathbf{4}} \times \mathbf{S}^{\mathbf{3}}=\left(S U(2)_{1} \times \mathbf{R}^{\geq 0}\right) \times \mathbf{S}^{\mathbf{3}}$ the type IIA manifold $\left(\mathbf{S}^{\mathbf{2}} \times \mathbf{R}^{\geq 0}\right) \times$ $\mathbf{S}^{\mathbf{3}}=\mathbf{R}^{\mathbf{3}} \times \mathbf{S}^{\mathbf{3}}$ with fixed point at the origin, i.e. the deformed conifold $T^{*} \mathbf{S}^{\mathbf{3}}$ with a D6-brane wrapping the zero-section (the cycle $\mathbf{S}_{\mathbf{A}}^{3}$ below). For $X_{2}$ or $X_{3}$ one gets $\mathbf{R}^{4} \times \mathbf{S}^{\mathbf{3}} / U(1)=\mathbf{R}^{4} \times \mathbf{S}^{2}$, the two small resolutions of the conifold together with a unit of RR two-form flux on $\mathbf{S}^{\mathbf{2}}$ (as $\mathbf{S}^{\mathbf{3}}$ is Hopf fibered by $\mathbf{S}_{\mathbf{1 1}}^{\mathbf{1}}$ over $\mathbf{S}^{\mathbf{2}}$ ). 
For the deformed conifold $T^{*} \mathbf{S}^{3}$ one has as relation between the type IIA (quaternionic) hypermultiplet modulus $Z$ (with complex part $z=\int_{Q} \Omega$, here $Q=A$ is the $\mathbf{S}^{3}$ ) and the $M$-theory variable $\Phi=\int_{Q} C+i \Upsilon$. Let $A$ and $B$ the compact and dual noncompact three-cycle, respectively: one has $A \simeq Q_{1}$ but $B=\mathbf{R}^{\mathbf{3}}=\mathbf{S}_{\mathbf{1}}^{\mathbf{3}} / U(1) \times \mathbf{R}^{\geq \mathbf{0}} \nsucceq$ $D_{1}=\mathbf{S}_{\mathbf{1}}^{3},\left(\mathbf{R}^{4}, D\right)$ corresponds after the circle-reduction to $\left(B, \mathbf{S}_{\mathbf{B}}^{2}\right) . A=Q$ is a supersymmetric cycle and $\left.\operatorname{Re} \Omega\right|_{A}=\operatorname{vol}_{\mathbf{S}^{\mathbf{3}}}=\left.\Upsilon\right|_{Q}$ and $\int_{A} \operatorname{Im} \Omega=0$. Further one has classically in $M$-theory that $\int_{D} C=0$, but $B \not D$ and rather $\int_{D} C=\int_{\mathbf{S}^{2}} B^{(2)}=\int_{\mathbf{R}^{3}} H^{(3)} \neq \int_{\mathbf{R}^{3}} C$ (the first equality by circle reduction of field and cycle). One may consider further the fate of the remaining $N=2$ parameter $\int_{B} C$ from the perspective of the additional $D 6$ brane on $\mathbf{S}_{\mathbf{Q}}^{3}$ which effects the reduction to $N=1$.

A local ${ }^{2}$ parameter on the corresponding (quantum) moduli space in $M$-theory is given by the membrane instanton amplitude $u=e^{i \Phi}$ (cf. app. A). The membrane instanton superpotential $W(u)$ is given [8] by the dilogarithm (cf. app. G)

$$
\operatorname{Li}(u)=\sum_{n \geq 1} \frac{u^{n}}{n^{2}}=-\int_{0}^{u} \log (1-u) d \log u
$$

There are two equivalent ways to express the monodromy. In a vector picture one assembles $L i$, the ordinary logarithm and the constants to a three-vector and analytic continuation along a loop $l_{i}$ in $\mathbf{P}^{1} \backslash\{0,1, \infty\}$ (encircling $z=i$ ) leads to the monodromy representation $M: \pi_{1}\left(\mathbf{P}^{\mathbf{1}} \backslash\{0,1, \infty\}\right) \rightarrow G l(3, \mathbf{C})$

$$
c_{3}=\left(\begin{array}{c}
\operatorname{Li}(u) \\
\log u \\
1
\end{array}\right): M\left(l_{0}\right)=\left(\begin{array}{ccc}
1 & 0 & 0 \\
0 & 1 & 2 \pi i \\
0 & 0 & 1
\end{array}\right), M\left(l_{1}\right)=\left(\begin{array}{ccc}
1 & -2 \pi i & 0 \\
0 & 1 & 0 \\
0 & 0 & 1
\end{array}\right)
$$

Alternatively, in a Heisenberg picture, consider the complexified Heisenberg group $\mathcal{H}_{\mathbf{C}}$ of upper triangular complex $3 \times 3$ matrices with 1's on the diagonal. Instead of $c_{3}$ one considers here the expression (a flat section of a suitable connection [8]) (cf. (G.16))

$$
\Lambda(u)=\left(\begin{array}{ccc}
1 & -\log \beta u & -\operatorname{Li}(u) \\
0 & 1 & \log u \\
0 & 0 & 1
\end{array}\right)
$$

and left operation with $\mathcal{H}_{\mathbf{z}}$ expresses the multi-valuedness (G.27), cf. footn. 4. More

\footnotetext{
${ }^{2}$ For comments on the somewhat non-standard technical sense of the notions 'modulus' and 'superpotential' caused by the non-compactness of $X_{7}$, and on the question of having a global coordinate on the quantum moduli space versus having a first order parameter, cf. [8].
} 
precisely one has for the monodromy along the loops $l_{i}$ representing left multipliers $h_{i}$

$$
h_{0}=\left(\begin{array}{ccc}
1 & 0 & 0 \\
0 & 1 & 2 \pi i \\
0 & 0 & 1
\end{array}\right) \quad, \quad h_{1}=\left(\begin{array}{ccc}
1 & 2 \pi i & 0 \\
0 & 1 & 0 \\
0 & 0 & 1
\end{array}\right)
$$

One has a real-valued non-holomorphic monodromy-invariant (i.e. single-valued) function

$$
\mathcal{L}(u)=\operatorname{Im} L i(u)-\operatorname{Im} \log \beta u \operatorname{Re} \log u
$$

These results arise as follows. One has from $(a, b \mid c) \rightarrow(x, y)=\left(e^{a}, e^{b}\right)$ a bundle over $\mathbf{C}_{\mathbf{x}}^{*} \times \mathbf{C}_{\mathbf{y}}^{*}$ with fibre ${ }^{3}(2 \pi i)^{2} \mathbf{Z} \backslash \mathbf{C}_{\mathbf{c}}$ (isomorphic to $\mathbf{C}^{*}$ via $c \rightarrow S:=e^{c / 2 \pi i}$ )

$$
\begin{gathered}
\mathcal{H}_{\mathbf{Z}} \backslash \mathcal{H}_{\mathbf{C}} \\
\downarrow \\
\mathbf{C}_{\mathbf{x}}^{*} \times \mathbf{C}_{\mathbf{y}}^{*}=(2 \pi i \mathbf{Z})^{2} \backslash \mathbf{C}_{\mathbf{a}, \mathbf{b}}^{\mathbf{2}}
\end{gathered}
$$

A pullback gives [8] the Heisenberg bundle $\underline{\mathcal{H}}$ over $\mathbf{P}^{\mathbf{1}} \backslash\{0,1, \infty\}$

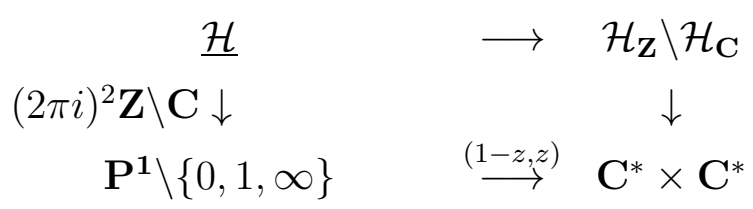

A section $s$ of $\underline{\mathcal{H}}$ has the form $s(z)=\mathcal{H}_{\mathbf{Z}}(-\log \beta z, \log z \mid c)$ and (2.3) is a flat section ${ }^{4}$. Heisenberg action for the universal hypermultiplet

For the universal hypermultiplet (cf. [14] - [17]) the quaternionic modulus consists of a complex part given by the four-dimensional dilaton-axion combination $e^{-2 \phi}+2 i D$ where the pseudoscalar $D$ arises by dualizing the external (Minkowski) component of $H_{3}=d B_{2}$

$$
H_{3}=e^{4 \phi} *_{4}(2 d D+i(\bar{c} d c-c d \bar{c}))
$$

together with a complex $C$-field period $c$

$$
C_{i j k}=\sqrt{2} c \Omega_{i j k}
$$

where we used the complex field

$$
S=e^{-2 \phi}+2 i D+c \bar{c}
$$

${ }^{3}$ the entries of $\mathcal{H}_{\mathbf{Z}}$ are actually from $\left((2 \pi i) \mathbf{Z},(2 \pi i) \mathbf{Z} \mid(2 \pi i)^{2} \mathbf{Z}\right)$; here $(a, b \mid c)$ gives the $g_{12}, g_{23}, g_{13}$ entry

${ }^{4}$ One finds [8] (undoing the fibre identification $c \rightarrow e^{c / 2 \pi i}=S$ ) that the flatness condition $d c=u d v$ just expresses the $L i$ integral, and that the coset takes into account the multi-valuedness (G.27). 
and the Kähler potential

$$
K=-\log (S+\bar{S}-2 c \bar{c})=2 \phi \quad-\log 2
$$

The Lagrange density for the scalar fields takes the form

$$
\begin{aligned}
\mathcal{L} & \sim(d \phi)^{2}+e^{2 \phi}|d c|^{2}+\frac{1}{2} e^{4 \phi}(2 d D+i(\bar{c} d c-c d \bar{c}))^{2} \\
& =K_{S \bar{S}} d S d \bar{S}+K_{S \bar{c}} d S d \bar{c}+K_{c \bar{S}} d c d \bar{S}+K_{c \bar{c}} d c d \bar{c} \\
=d s^{2} & =e^{2 K}(d S d \bar{S}-2 c d S d \bar{c}-2 \bar{c} d c d \bar{S}+2(S+\bar{S}) d c d \bar{c})
\end{aligned}
$$

The mentioned line element for the space spanned by the parameters $S$ and $c$ gives the quaternionic space $S U(2,1) / U(2)$. The classical Lagrangian has eight symmetries (the left $S U(2,1)$ action), four of which (related to scale transformations and further isometries) are not preserved in the quantum theory. By contrast from the remaining four symmetries one gets symmetries even present when the membrane and fivebrane instantons are taken into account, broken to their discrete parts [14]. This leads first to the $\mathbf{Z}_{\mathbf{2}}$ generated by the involution

$$
\iota: \operatorname{Re} c \longleftrightarrow \operatorname{Im} c
$$

Then there is a group of isometries associated with the following shifts $(\alpha, \beta, \gamma \in \mathbf{R}, \delta=$ $\gamma+i \beta$; note that, like the action of $\iota$, this leaves $K$ invariant)

$$
\left(\begin{array}{c}
S \\
c \\
1
\end{array}\right) \longrightarrow\left(\begin{array}{ccc}
1 & 2 \delta & \delta \bar{\delta}+i \alpha \\
0 & 1 & \bar{\delta} \\
0 & 0 & 1
\end{array}\right)\left(\begin{array}{c}
S \\
c \\
1
\end{array}\right)
$$

Note that when just turning on an individual parameter $\alpha, \beta, \gamma$, leading to corresponding transformations $T_{\alpha}, T_{\beta}, T_{\gamma}$, one has the characteristic relation $T_{\beta} T_{\gamma}=T_{\alpha=4 \beta \gamma} T_{\gamma} T_{\beta}$. If $Z$ denotes the corresponding group with integral entries (enhanced with $\iota$ ) then the moduli space is actually $\mathcal{M}=Z \backslash S U(2,1) / U(2)$. Note the invariant expression (cf. (2.5))

$$
e^{-2 \phi}=\frac{1}{2} e^{-K}=\operatorname{Re} S-c \bar{c}
$$

As in (2.3) one may consider the action not on the vector $(S, c, 1)^{t}$ but on a Heisenberg group element; for this consider the rescaled coordinate $e:=\sqrt{2} c, \eta:=\sqrt{2} \delta$

$$
\left(\begin{array}{ccc}
1 & \bar{e} & S \\
0 & 1 & e \\
0 & 0 & 1
\end{array}\right) \longrightarrow\left(\begin{array}{ccc}
1 & \eta & \frac{\eta \bar{\eta}}{2}+i \alpha \\
0 & 1 & \bar{\eta} \\
0 & 0 & 1
\end{array}\right)\left(\begin{array}{ccc}
1 & \bar{e} & S \\
0 & 1 & e \\
0 & 0 & 1
\end{array}\right)
$$


with the invariant expression $2 e^{-2 \phi}=e^{-K}=(S+\bar{S})-e \bar{e}$.

Note further that one has a contribution to the $d c d \bar{c}$ component of the metric as given by the following term in the action (up to the $e^{4 \phi}$ normalization factor)

\section{$2 \operatorname{Re} S d c d \bar{c}$}

Associated with the three isometries are classically conserved Noether currents, first $J_{\alpha}=2 * H_{3}$ with the corresponding conserved fivebrane charge $Q_{\alpha}=\int_{\mathcal{C}_{3}} * J_{\alpha}=2 \int_{\mathbf{S}^{3}} H_{3}$ for an euclidean fivebrane wrapping the whole $X$, being surrounded by an $\mathbf{S}^{\mathbf{3}}$ in the transversal non-compact $\mathbf{R}^{4}$. More important for us are the other two currents

$$
\begin{aligned}
& J_{\beta}=2 i e^{K} d(c-\bar{c})+2(c+\bar{c}) J_{\alpha} \\
& J_{\gamma}=2 e^{K} d(c+\bar{c})-2 i(c-\bar{c}) J_{\alpha}
\end{aligned}
$$

where the corresponding conserved charges

$$
Q_{\beta, \gamma}=\int_{\mathcal{C}_{3}} * J_{\beta, \gamma}
$$

correspond to membrane charges ${ }^{5}$

$$
\int_{\mathcal{C}_{6}} e^{\phi / 2} *_{10} d C+C \wedge H_{3}
$$

Compare in the $M$-theory conifold case the quantities $\int_{\mathbf{S}_{\mathbf{Q} / \mathbf{D}}^{3}} *_{7} G$. In the scalar potential term ${ }^{6} \int_{X}|G|^{2}=\int_{B} G \cdot \int_{Q} *_{7} G \leftrightarrow \int_{D} C \cdot \int_{Q} *_{7} G$ they accompany (the imaginary parts of) $\log \eta=\log \beta u$ and $\log u$ which occupy the $\Lambda_{12}, \Lambda_{23}$ places in (2.3) just as do the membrane parameters $\beta, \gamma$ (which go with $Q_{\beta \gamma}$ ) in (2.14) (a $\mathbf{Z}_{\mathbf{2}}$ exchanges $Q$ and $D$; cf. $\iota$ above).

One may parametrize the coset space $\mathcal{M}_{U H}=S U(2,1) / U(1)$ also by two complex scalars $z_{1}$ and $z_{2}$ in the open four-ball $\left|z_{1}\right|^{2}+\left|z_{2}\right|^{2}<1$ (cf. app. B) which relate to $S$ and $c$ by $S=\frac{1-z_{1}}{1+z_{1}}, c=\frac{z_{2}}{1+z_{1}}$; introduction of polar coordinates (B.3) gives then a $T^{2}=\Lambda \backslash \mathbf{C}$ fibration $^{7}$ over the open quarter-disc $\mathcal{D}$ parametrised by $z:=\left|z_{1}\right|+i\left|z_{2}\right|$

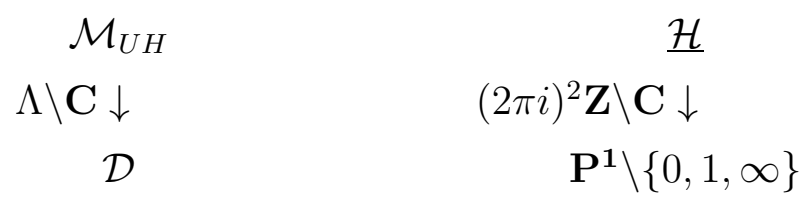

where we contrasted with the Heisenberg bundle (in principle we could also consider on the lhs the actual moduli space after modding by the left integral shifts).

\footnotetext{
${ }^{5}$ Note that, very schematically, $J_{\beta / \gamma} \sim d c+c \cdot *_{4} H_{3}$ and so $*_{4} J_{\beta / \gamma} \sim *_{4} d c+c \cdot H_{3}$ and $(d C)_{\mu i j k} \sim$ $\partial_{\mu} c \epsilon_{i j k}$ by (2.9), so for this component $*_{10}(d C) \sim *_{4} \partial_{\mu} c *_{6} \epsilon_{i j k}=*_{4} \partial_{\mu} c \epsilon_{i \bar{j} \bar{k}}$; the $c \cdot H_{3}$ matches as well.

${ }^{6}$ either for a flux or in a two-point function with an auxiliary $G$ to evaluate $W_{\text {mem }}$ [8]

${ }^{7}$ the lattice $\Lambda$ occurring here is not to be confused with the flat section (2.3)
} 


\section{Remark 1: Metric corrections}

Euclidean D2-branes wrapped on $\mathbf{S}_{\mathbf{A}}^{3}$ lead to metric corrections of the hypermultiplet moduli space [2], [3], [13]. We relate this to a twisted dilogarithm. The corrections were obtained approaching the conifold point $z \rightarrow 0$ in a suitable limit to keep the result independent of the details of the embedding of the local conifold $T^{*} \mathbf{S}^{3}$ in a specific global Calabi-Yau model. The size $|w|=e^{-\rho}$ of the rescaled variable $w$ ( $\lambda$ the string coupling)

$$
\frac{z}{\lambda}=: w=e^{i(\theta+i \rho)}
$$

was kept fixed. With $x=\int_{A} C, t=\int_{B} C$ the metric (in the string frame) is

$$
d s^{2}=V^{-1}\left(d t-\left(A_{x} d x+A_{w} d \bar{w}+A_{\bar{w}} d w\right)\right)^{2}+V\left(d x^{2}+d w d \bar{w}\right)
$$

where the scalar potential $V$ and the vector potential $A$ with $\nabla V=\nabla \times A$ are

$$
\begin{aligned}
V & =\frac{1}{4 \pi} \log \left(\frac{\Lambda^{2}}{|w|^{2}}\right)+\frac{1}{2 \pi} \sum_{n \neq 0} e^{2 \pi i n x} K_{0}(2 \pi|n||w|) \\
A_{x} & =-\frac{1}{2 \pi} \theta, A_{w}=A_{\bar{w}}=\frac{1}{2 \pi i} \sum_{n \neq 0} \operatorname{sign}(n) e^{2 \pi i n x} K_{1}(2 \pi|n||w|)
\end{aligned}
$$

To make the instanton contributions manifest expand the Bessel function $K_{0}$ (large $|w|$ )

$$
V \sim \frac{1}{4 \pi} \log \left(\frac{\Lambda^{2}}{|w|^{2}}\right)+\frac{1}{\pi} \sum_{n>0} \frac{1}{\sqrt{n|w|}} \cos (2 \pi n x) e^{-2 \pi n|w|}
$$

In the appropriate variable (so the $n$-instanton action is $S_{n}=n|w|$ ) (cf. the multiplet reduction discussed earlier, restricted to $Q$ one has $\Omega=\operatorname{Re} \Omega=|\Omega|=v o l$ )

$$
u_{\phi}:=e^{2 \pi i \phi}=e^{-2 \pi|w|+2 \pi i x}, \quad \phi:=\int_{Q} C+i \frac{1}{\lambda} \Omega=x+i|w|=x+i e^{-\rho}
$$

this leads to an instanton contribution to the $d \rho^{2}$ component of the metric as given by the following terms in the effective action ${ }^{8}$ (up to a $1 / 2 \pi$ normalization factor)

$$
2 \operatorname{Re} L\left(u_{\phi}\right) d \rho d \rho=2 \operatorname{Re}\left(\sum_{n>0} \frac{u_{\phi}^{n}}{n^{2}} S_{n}^{3 / 2}\right) d \rho d \rho=4 \frac{1}{|w|^{1 / 2}} \operatorname{Re}\left(\sum_{n>0} \frac{u_{\phi}^{n}}{n^{2}} n^{3 / 2}\right) d w d \bar{w}+\ldots
$$

This shows a 'twisted' version $L(u):=\left(-\frac{1}{2 \pi}\right)^{3 / 2} \sum_{n>0} \frac{u^{n}}{n^{2}}(n \operatorname{Re} \log u)^{3 / 2}$ of the superpotential $W=L i(u)$ from membrane instantons wrapped on $\mathbf{S}_{\mathbf{A}}^{3}$ (cf. (1.1)), with the appropriate ${ }^{9}$ power of $S_{n}$ extracted. As one has the same form for the $d x^{2}$ component recall that (2.17) gave $2 \operatorname{Re} S d c d \bar{c}$ and (2.14) showed $S$ in a position analogous to $\operatorname{Li}\left(e^{c}\right)$ (we had here the $e^{2 \pi i x}$ in $u_{\phi}$; still note the quaternionic vs. hyperkähler difference).

\footnotetext{
${ }^{8}$ keeping only the leading contribution (not from $A_{z}$ expansion) to the $n$-instanton term

${ }^{9}$ the extracted universal power $S_{n}^{3 / 2}$ explains itself according to [13] as $3 / 2=p+a$ where $p=2$ is the number of fields in the kinetic term and $a=4\left(\frac{1}{4}-\frac{3}{8}\right)$ should emerge form the Jacobian for the change of variables from zero modes to collective coordinates and a weight $1 / 4$ and $-3 / 8$ is associated to each bosonic and fermionic collective coordinate, respectively (the hypermultiplet contains four real scalars)
} 


\section{Remark 2: The conifold monodromy}

Consider the moduli space singularity caused by vanishing of the $\mathbf{S}^{\mathbf{3}}$ of the deformed conifold at $z=\operatorname{vol}\left(\mathbf{S}_{\mathbf{A}}^{\mathbf{3}}\right) \rightarrow 0$ and its associated monodromy. This is usually discussed in the framework of the type IIB vector multiplet moduli space. We wish to compare some of these type II(B) relations with $M$-theory relations.

First, concerning type IIB note that for the deformed conifold $x^{2}+y^{2}+z^{2}+v^{2}=\mu$ with $\Omega \sim \frac{d x d y d z}{v}=\frac{d x d y d z}{\sqrt{\mu-x^{2}-y^{2}-z^{2}}}$ and the three-cycles $A, B$ seen as $\mathbf{S}^{2}$ 's (spanned by $\operatorname{Re} y, \operatorname{Re} z$ ) over $x$ one has (cf. [5])

$$
\int_{\mathbf{S}^{2}} \Omega \sim d x \sqrt{x^{2}-\mu}
$$

and finds then (the last equality up to $\mathcal{O}\left(\frac{1}{\sqrt[3]{c}}\right)$ correction)

$$
\begin{aligned}
z=\int_{A} \Omega & =\int_{A} \operatorname{Re} \Omega+i \int_{A} \operatorname{Im} \Omega=\int_{A} v o l=\frac{1}{2 \pi i} \int_{-\sqrt{\mu}}^{\sqrt{\mu}} d x \sqrt{x^{2}-\mu}=\mu / 4 \\
F_{z}=\Pi=\int_{B} \Omega & =\frac{1}{2 \pi i} \int_{\sqrt{\mu}}^{\sqrt{c}} d x \sqrt{x^{2}-\mu} \approx \frac{1}{2 \pi i}\left(\frac{1}{2} c-z(\log c+1-\log z)\right)
\end{aligned}
$$

where $B \rightarrow B-A$, or $\Pi \rightarrow \Pi-z$, corresponds to $c \rightarrow e^{2 \pi i} c$.

Now compare the $M$-theory relations. $Q \simeq A$ but, as remarked already above, $D \nsucceq B$ : the $D$ is an $\mathbf{S}^{3}$ at some $r$, whereas $B \cong \mathbf{R}^{3}$ is the cone over $\mathbf{S}^{2}$ (which may be truncated with a cut-off at $c$ ). Usually the relations to follow are considered [1], [8] at the semicalssical end $P_{i}$ corresponding to $\int_{Q} \Upsilon \approx \infty$ or $u_{i} \approx 0$. By contrast we are now, in classical terms, near a phase transition where $\operatorname{vol}(Q) \rightarrow 0$ (i.e. one has reached the 'critical circle' $\left|u_{i}\right|=1[8]$ ), so we are looking near $P_{i+1}$ but still in variables properly adapted to $P_{i}$. Nevertheless note that (with $z_{i}:=\log u_{i}$ )

$$
\begin{aligned}
\zeta_{i}:=\operatorname{Re} z_{i} & =\int_{Q_{i}}-\Upsilon=-2 \pi^{2} r_{0}^{3} \\
\xi_{i} & :=\operatorname{Re} x_{i}=\int_{D_{i}} \text { vol } \approx r^{3}+\text { const } \cdot f_{i} r_{0}^{3}=r^{3}+\text { const }^{\prime} \cdot \operatorname{Re} \log y_{i} \operatorname{Re} z_{i}
\end{aligned}
$$

Do they reflect an analogue of $\Pi \sim z \log z$ in (2.30) for $\int_{B} \Omega$ (turned off all $C$ field integrals) ? Now note that at $P_{i}$ one has volume defects $\left(f_{i-1}, f_{i}, f_{i+1}\right) \sim \rho(1,-2,1)$ with $\rho \rightarrow \infty$ (cf app. A, [1], [8]). Therefore $\operatorname{Re} \log y_{i}=f_{i}$ and $\operatorname{Re} \log \eta_{i}=\left(f_{i-1}-\right.$ $\left.f_{i+1}\right) / 3$ behave identically near $P_{i+1}$. From this relation $\operatorname{Re} \log y_{i} \sim \operatorname{Re} \log \eta_{i}$ and the interpretation $\beta u_{i} \sim \eta_{i}$ (cf. [8]) one has with $\beta u_{i}=\frac{1}{1-e^{z_{i}}} \approx \frac{1}{-z_{i}}$ that $\log z_{i} \sim \log y_{i}$ holds; so (2.31) gives indeed $\xi_{i} \sim \zeta_{i} \log \zeta_{i}$ (near $P_{i+1}$ we are at $z_{(i)} \approx 0 \leftrightarrow u_{i} \approx 1 \leftrightarrow \beta u_{i} \approx \infty$, so according to $y_{i}=\eta_{i+1} / \eta_{i-1} \approx 1 / 0 \approx \infty \approx \eta_{i}$ ). 


\section{Remark 3: Instanton interpretation of the Schwinger computation}

The classical non-supersymmetric Schwinger computation has $N=2$ and $N=1$ avatars (cf. Introduction and app. $\mathrm{F})^{10}$. We point here to relations between even the way of derivation of the membrane instanton superpotential (1.1) and an instanton reinterpretation [12] of the occurrence of $L i$ in the classical computation. The latter gives with

$$
\tau=i \mu \quad, \quad \mu=\frac{m^{2}}{2 e} \frac{1}{E}
$$

(we assume $e E=|e||E|$ ) for pair creation in a constant background electric field $E$ an imaginary energy density (giving the probability density) (for background cf. [11] )

$$
w \sim \operatorname{Li}\left(e^{2 \pi i \tau}\right)
$$

The one-loop effective action for electrons in a background electro-magnetic field is in the case of a static magnetic field (with $\not D:=\gamma^{\nu}\left(\partial_{\nu}+i e A_{\nu}\right), B_{n}$ the Bernoulli numbers)

$$
\begin{aligned}
S_{\text {eff }} & =-i \log \operatorname{det}(i \not D-m)=-\frac{i}{2} \log \operatorname{det}\left(\not D^{2}+m^{2}\right)=\frac{i}{2} \int_{0}^{\infty} \frac{d s}{s} \operatorname{tr} e^{-s\left(\not D^{2}+m^{2}\right)} \\
& =-\frac{e^{2} B^{2}}{8 \pi^{2}} \int_{0}^{\infty} \frac{d s}{s^{2}}\left(\operatorname{coth} s-\frac{1}{s}-\frac{s}{3}\right) e^{-\frac{m^{2}}{e B}} \\
& =\frac{e^{2} B^{2}}{2 \pi^{2}} T V \sum_{n=1}^{\infty} \frac{B_{2 n+2}}{(2 n+2)(2 n+1) 2 n}\left(\frac{2 e B}{m^{2}}\right)^{2 n}
\end{aligned}
$$

(in the case of a static magnetic field this represents the negative energy of the electrons in the background; the subtractions of $\frac{1}{s}$ and $\frac{s}{3}$ correspond to the subtraction of the zero-field contribution and a logarithmic charge renormalization, respectively).

In the case of a static uniform electric field one gets (from $B \longrightarrow i E$ ) a corresponding perturbative series but now the effective action is complex with its imaginary part (a sum over non-perturbative tunneling amplitudes; this comes from the poles which have moved onto the contour of integration, the real part is the remaining regulated principal part) giving the pair-production rate

$$
\begin{aligned}
S_{\text {eff }} & =\frac{e^{2} E^{2}}{8 \pi^{2}} T V \int_{0}^{\infty} \frac{d s}{s^{2}}\left(\cot s-\frac{1}{s}+\frac{s}{3}\right) e^{-2 \mu s} \\
& =\frac{e^{2} E^{2}}{8 \pi^{3}} T V\left(-4 \pi \sum_{n=1}^{\infty} \frac{B_{2 n+2}}{(2 n+2)(2 n+1) 2 n} \frac{1}{\tau^{2 n}}+i \operatorname{Li}\left(e^{2 \pi i \tau}\right)\right)
\end{aligned}
$$

\footnotetext{
${ }^{10}$ Note that in the framework of $N=2$ supersymmetry the original non-supersymmetric Schwinger computation of a vacuum amplitude (for the contribution of a scalar particle) is transformed to a computation of an $R^{2}$ correction from contributions of BPS states given by D0-branes and wrapped D2-branes on an isolated $\mathbf{S}^{2}$, say, (bound states of the lightest states at strong coupling, corresponding to the $M$-theory 'lift' where such a bound state corresponds to a M2-brane with momentum around the $\mathbf{S}_{\mathbf{1 1}}^{\mathbf{1}}$ ) as the spin content compensates for the extra insertions of the curvature.
} 
It is instructive to compare the analogies to our situation. Instead of the proper time formalism describing the Dirac fermion in the constant uniform electric field one can consider an equivalent one-dimensional static non-relativistic harmonic oscillator [12]. In the (bosonic) Fourier-mode $\Phi(t, \vec{x})=e^{i\left(\mathbf{k}_{\perp} \mathbf{x}_{\perp}-\omega t\right)} \phi_{\omega, \mathbf{k}_{\perp}}\left(x_{\|}\right)$one has for the tunnel process wave function $\phi_{\omega, \mathbf{k}_{\perp}}\left(x_{\|}\right) \sim D\left(a_{\mathbf{k}_{\perp}}, \xi\right)$ with $D$ a parabolic cylinder function and

$$
\begin{gathered}
\xi=\sqrt{2 / e E} x \quad, \quad x:=\omega+e E x_{\|} \\
a_{\mathbf{k}_{\perp}}=\frac{\mu}{2 e E} \quad, \quad \mu:=m^{2}+\mathbf{k}_{\perp}{ }^{2}
\end{gathered}
$$

The one-instanton action is an integral between the classical turning points $x_{ \pm}= \pm \sqrt{\mu}-\omega$

$$
S_{\mathbf{k}_{\perp}}=i \int_{x_{-}}^{x_{+}} d x_{\|} \sqrt{x^{2}-\mu}=\pi a_{\mathbf{k}_{\perp}}
$$

Now the tunneling and the no pair-production probability for a fermion are

$$
P_{\mathbf{k}_{\perp}}^{\text {tunnel }}=e^{-2 S_{\mathbf{k}_{\perp}}} \quad, \quad P_{\mathbf{k}_{\perp}}^{\text {no pair }}=1-e^{-2 S_{\mathbf{k}_{\perp}}}
$$

With $<0$, out $\mid 0$, in $>=e^{i \int d^{4} x \mathcal{L}}$ one has as vacuum-to-vacuum transition amplitude $P_{0}$

$$
e^{- \text {total pair production }}=\prod_{\text {all states }} P_{\mathbf{k}_{\perp}}^{\text {no pair }}=\mid<0, \text { out } \mid 0, \text { in }>\left.\right|^{2}=e^{-2 V T \operatorname{Im} \mathcal{L}}
$$

Here $\mathcal{L}$ is the effective Lagrangian: the dispersive real part includes the non-linear terms in the Euler-Heisenberg Lagrangian, the absorptive imaginary part describes the pair production $\operatorname{Im} S_{\text {eff }}$ in (2.35). For fermions to be produced $P_{0}$ should have a complex phase so that $\left|P_{0}\right|^{2}<1$. One gets as pair production rate per unit volume and unit time in $\mathbf{R}^{1, d}$ (using $\int d \omega=e E V_{\|}, V=V_{\perp} V_{\|}$)

$$
\begin{aligned}
w & =2 \operatorname{Im} \mathcal{L}=\frac{1}{V T} \sum_{\text {all states }} \log \left(1-e^{-2 S_{\mathbf{k}_{\perp}}}\right)=\frac{2 V_{\perp}}{V} \int \frac{d \omega}{2 \pi} \frac{d \mathbf{k}_{\perp}{ }^{d-1}}{(2 \pi)^{d-1}} \sum_{n=1}^{\infty} \frac{1}{n} e^{-2 \pi n \mu} \\
& =\frac{2}{(2 \pi)^{d}} \sum_{n=1}^{\infty} \frac{e E}{n}\left(\frac{e E}{n}\right)^{\frac{d-1}{2}} e^{-\pi n m^{2} / e E}=\frac{2(e E)^{(d+1) / 2}}{(2 \pi)^{d}} L i_{(d+1) / 2}\left(e^{\tau}\right)
\end{aligned}
$$

Now, the point of this remark is that the modulus and dilogarithm even arise in this classical case, when interpreted this way as an instanton calculation, the same way as in the membrane instanton case. For notice that the instanton action (2.37) resembles the way $\operatorname{vol}\left(\mathbf{S}_{\mathbf{Q}}^{\mathbf{3}}\right)$ occurs in (2.29) and that even the way $L i_{2}$ arises is identical; for the difference between the determined integral over the momenta in (2.40) and the undetermined integral in $\operatorname{Li}\left(e^{x}\right)=\int_{-\infty}^{x} d y \log \beta e^{y}$ is only apparent (let $A=\pi / e E$ ): $w \sim \int_{0}^{\infty} r d r \log \left(1-e^{-A\left(m^{2}+r^{2}\right)}\right) \sim \int_{0}^{\infty} d \rho \log \left(1-e^{-A\left(m^{2}+\rho\right)}\right)=\int_{m^{2}}^{\infty} d \sigma \log \left(1-e^{-A \sigma}\right)=$ $\frac{1}{A} \int_{-\infty}^{-A m^{2}} d \chi \log \left(1-e^{\chi}\right)=-\frac{1}{A} \operatorname{Li}\left(e^{2 \pi i \tau}\right)$. The analogy should extend to a full $\tau \in \mathbf{C}$. 


\section{The flat bundle of five-dimensional $N=2 S U(2)$ field theory}

The period $A_{D}$ of $5 \mathrm{D} N=2 S U(2)$ field theory is given by $L i\left(e^{A}\right)$ (cf. (3.13)), the $4 \mathrm{D}$ period $a_{D}$ relates to the conifold (at the monopole point $\left.\tilde{u}=1\right)^{11}: \Xi_{6} \sim a_{D} \sim x_{+} \sim \tilde{u}-1$.

4D $N=2$ gauge theories arise from type IIA string theory on a Calabi-Yau $X$. As type IIA is $M$-theory reduced on a circle, the $4 \mathrm{D}$ gauge theory is coming from a $5 \mathrm{D}$ gauge theory, given by $M$-theory on $X \times \mathbf{S}_{\mathbf{R}}^{1}$ with $R=g_{s}^{2 / 3} l_{p, 11}$. Then a reinterpretation of the $4 \mathrm{D}$ (vector multiplet) prepotential becomes possible. The latter consists of a piecewise cubic polynomial part (in the scalar vev's of the vecor multiplets) plus instanton corrections (suppressed in the 5D decompactification limit). The world-sheet instanton corrections are reinterpreted as one-loop corrections in the 5D space-time $\mathbf{R}^{4} \times \mathbf{S}_{\mathbf{R}}^{1}$.

The prepotential $\mathcal{F}_{R}$ of $M$-theory on ${ }^{12} X \times \mathbf{S}_{\mathbf{R}}^{1}$ has the cubic form as $5 \mathrm{D}$ limit $(R \rightarrow \infty)$ and $\mathcal{F}_{4 D} \sim \frac{1}{2} T^{2}\left(\log \left(\frac{T}{\Lambda}\right)^{2}+\sum_{n=1}^{\infty} \frac{c_{n}}{(T / \Lambda)^{4 n}}\right)$ as 4 D limit $(R \rightarrow 0)$ which shows the instanton corrections ${ }^{13}$. $R \sim \epsilon \rightarrow 0$ induces the scaling limit (E.4) by $\Lambda_{U V} \approx \frac{1}{R_{11}}=\frac{1}{g_{s} l_{s}}$ and (E.6). Now even before taking the scaling limit one can interprete [27] the world-sheet instanton sum (E.3) as a perturbative field-theoretic one-loop correction in $\mathbf{R}^{4} \times \mathbf{S}_{\mathbf{R}}^{1}{ }^{14}$

To substantiate the interpretation take the weak coupling limit $T_{b} \rightarrow \infty$ (without $T_{f} \rightarrow 0$ ); so instantons wrapping the base decouple and with $c_{0, m}=-2 \delta_{m, 1}$ one finds

$$
\tau_{f}=2 \log \left(1-e^{-T_{f}}\right)
$$

When $T_{f} \rightarrow 0$ one gets with $\tau_{f} \sim 2 \log M_{W}$ (from $T_{f} \sim M_{W}$ ) the expected gauge theory correction $^{15}$. However, before taking this limit $T_{f} \rightarrow 0$ one finds from (3.1)

$$
\tau_{f}=-T_{f}+\log \sinh ^{2} \frac{T_{f}}{2}
$$

(up to $2 \log 2$; cf. (3.19)). By $\frac{1}{g_{4}^{2}} \sim \frac{2 \pi R}{g_{5}^{2}}$, i.e. $\tau_{5} \sim \frac{\tau_{4}}{2 \pi R}$ this gives back the $5 \mathrm{D}$ term (3.4) via $T_{f}=2 \pi R M_{W}$ (up to the linear piece, the decompactification limit, cf. footn. 22,40 ).

\footnotetext{
${ }^{11} \Xi_{6}$ the period of the 6 -cycle in type IIA, or the vanishing $\mathbf{S}^{\mathbf{3}}$ of the conifold in type IIB, cf. (D.15), (E.9); for the string/ $M$-theoretic embedding of the 4D/5D field theories cf. app. D, E

${ }^{12}$ The vector multiplets of the $5 \mathrm{D}$ gauge theory given by $M$-theory on $X$ itself are given (up to the graviphoton) by sizes of two-cycles, the prepotential is $\mathcal{F}=\frac{1}{6} \sum C_{i j k} T_{i} T_{j} T_{k}$ (in a Kähler cone) where $C_{i j k}$ are the classical intersection numbers of the divisors $i, j, k$ and the $T^{i}$ the areas of the dual two-cycles.

${ }^{13} \mathrm{~T}$ a complex scalar of a $4 \mathrm{D}$ vector built by a real scalar of a $5 \mathrm{D}$ vector and an $\mathbf{S}^{\mathbf{1}}$ Wilson loop

${ }^{14}$ The Gromov-Witten invariants correspond to contributions of 5D BPS states (weighted according to spin) given by membranes wrapped on holomorphic curves of classes $n b+m f \in H_{2}\left(\mathcal{N}\left(F_{0}\right), \mathbf{Z}\right)$ of central charge $Z_{n, m} \sim \frac{n T_{b}+m T_{f}}{g_{s} l_{s}}$ and mass $M_{n, m} \sim\left|Z_{n, m}\right| ; c_{n, m}$ is the Euler characteristic of the moduli space $\mathcal{M}_{n, m}$, i.e. the 'number' of curves of degree $(n, m)$ taking into account spin factors.

${ }^{15}$ perturbative one-loop contribution; to get the field theory instantons one needs the double scaling
} 


\subsection{The flat bundle of the five-dimensional field theory}

At a generic point of the vector multiplet moduli space the gauge group is broken to a product of $U(1)$ 's. The gauge coupling $\tau=\frac{4 \pi i}{g^{2}}$ of such a $U(1)$ gets one-loop corrections from particles charged under this $U(1)$ (like the $W$-bosons) which have in $\mathbf{R}^{\mathbf{5}}$ the form

$$
e^{2} \int \frac{d^{5} k}{\left(k^{2}+M^{2}\right)^{2}} \sim e^{2} M
$$

(up to a divergent constant; $e, M$ the charge and mass of the particle). On $\mathbf{R}^{4} \times \mathbf{S}_{R}^{1}$ one has further corrections from world-line instantons of the particle around the $\mathbf{S}^{\mathbf{1}}$

$$
M+2 \sum_{k=1}^{\infty} \frac{1}{2 \pi R k} e^{-2 \pi R k M}=\frac{1}{2 \pi R} \log \sinh ^{2} \pi M R
$$

with $2 \pi R k M$ the action of a world-line k-instanton (properly speaking one has to write $|M|$ as the mass which was in 5D the real scalar component of a vector multiplet has become complex after compactifying on a further $\mathbf{S}^{\mathbf{1}}$ by combining with the Wilson loop).

Let us introduce besides the ordinary quantities $U, A, A_{D}$ of the five-dimensional theory the following expressions which are rescaled to dimensionless combinations

$$
\mathcal{U}=R^{2} U, \mathcal{A}=R A, \mathcal{A}_{D}=R A_{D}, \zeta=\Lambda R
$$

The $S U(2)$ curve for the Coulomb branch of the $N=2$ theory on $\mathbf{R}^{\mathbf{4}} \times \mathbf{S}_{R}^{1}$ is ([26] - [29])

$$
y^{2}=\left(x^{2}-\Lambda^{4}\right)\left(x-\frac{1}{2 R^{2}}\left(\mathcal{U}^{2}-1\right)\right)
$$

This corresponds ${ }^{16}$ with the four-dimensional Seiberg/Witten curve (D.1) under

$$
u=\frac{1}{2 R^{2}}\left(\mathcal{U}^{2}-1\right)=\frac{1}{2}\left(R^{2} U^{2}-\frac{1}{R^{2}}\right)
$$

From the $4 \mathrm{D}$ relation $\Delta_{4}=4 R^{4}\left(\Lambda^{4}-u^{2}\right)$ one finds for the 5D discriminant

$$
\Delta=(2 \zeta)^{2}-\left(1-\mathcal{U}^{2}\right)^{2} \quad\left(\stackrel{\Lambda \rightarrow 0}{\longrightarrow}-\left(1-\mathcal{U}^{2}\right)^{2}\right)
$$

The relevant one-form is $\frac{d \lambda_{5}}{d U}=c \frac{d x}{y}$ (with $c=\sqrt{2} /(8 \pi)$ ). The period integrals of the curve

$$
\frac{d A}{d U}=c \oint_{\alpha} \frac{d x}{y}, \frac{d A_{D}}{d U}=c \oint_{\beta} \frac{d x}{y}
$$

relate to the field-theoretical quantities ${ }^{17}$. The effective coupling is given by

$$
\tau_{5}=\frac{d A_{D}}{d A}=\frac{d A_{D} / d U}{d A / d U}
$$

\footnotetext{
${ }^{16} \mathrm{~A}$ quartic form $y^{2}=\left(x^{2}+s x+1\right)^{2}-4 \zeta^{4}$ is also used which corresponds to the quartic form $\tilde{y}^{2}=\left(\tilde{x}^{2}-u\right)^{2}-\Lambda^{4}$ of the $4 \mathrm{D}$ curve (footn. 27$)$ via $\sqrt{2} R \tilde{x}=x+s / 2,2 R^{2} \tilde{y}=y,-U=s / 2$ (by (3.7))

${ }^{17}$ one has the compatibility relations $\frac{d \lambda_{5}}{d U}=\frac{d \lambda_{4}}{d u}$ and $\frac{d A}{d U}=\frac{d a}{d u}, \frac{d A_{D}}{d U}=\frac{d a_{D}}{d u}$
} 
In the weak coupling limit $(\mathcal{U} \approx \infty$, corresponding to the $4 \mathrm{D}$ weak coupling limit $u \approx \infty)$ one gets (cf. $(3.24)$ ) (in $\tilde{\mathcal{A}}_{D}=2 \mathcal{A}_{D}, \tilde{\mathcal{A}}=-4 \mathcal{A}$ and for the monodromy around $\mathcal{U} \approx \infty$ )

$$
\left(\begin{array}{c}
\tilde{\mathcal{A}_{D}} \\
\tilde{\mathcal{A}} \\
1
\end{array}\right)=\left(\begin{array}{ccc}
1 & \log \zeta & 0 \\
0 & 1 & 0 \\
0 & 0 & 1
\end{array}\right)\left(\begin{array}{c}
\operatorname{Li}(x) \\
\log x \\
1
\end{array}\right), \quad \tilde{M}_{\infty}=\left(\begin{array}{ccc}
1 & -2 \cdot 2 \pi i & 2 \pi i \log \zeta \\
0 & 1 & 2 \cdot 2 \pi i \\
0 & 0 & 1
\end{array}\right)
$$

Compare with the result that one has by (2.2) with $M\left(l_{\infty}\right)=M\left(l_{0}\right)^{-1} M\left(l_{1}\right)^{-1}$

$$
c_{3}=\left(\begin{array}{c}
\operatorname{Li}(y) \\
\log y \\
1
\end{array}\right) \quad, \quad M\left(l_{\infty}\right)=\left(\begin{array}{ccc}
1 & 2 \pi i & 0 \\
0 & 1 & -2 \pi i \\
0 & 0 & 1
\end{array}\right)
$$

So one has that the $5 \mathrm{D}$ analogue $\mathcal{A}_{D}$ of the conifold period $a_{D}$

$$
\tilde{\mathcal{A}}_{D}=\operatorname{Li}\left(e^{\tilde{\mathcal{A}}}\right)+(\log \zeta) \tilde{\mathcal{A}}
$$

is given by the dilogarithm ${ }^{18}$ of (exp of) $\mathcal{A}$ up to a linear modification (cf. [8], sect. 4.4).

Note that the object $\underline{A}:=\left(\begin{array}{c}A_{D} \\ A \\ m_{R}\end{array}\right)$ with the mass scale $m_{R}:=1 / R$ also has the monodromy (3.24) around $U \approx \infty$. By $\operatorname{contrast}^{19}$, for the four-dimensional theory with one massive hypermultiplet one has the monodromy (D.14) around $a_{0}$.

\section{computational details}

To get these results note that the periods (3.9) fulfill the Picard-Fuchs equation ${ }^{20}$

$$
\left[\partial_{\mathcal{U}}^{2}+\frac{1}{\mathcal{U}}\left(\frac{4 \mathcal{U}^{2}}{\Delta}\left(1-\mathcal{U}^{2}\right)-1\right) \partial_{\mathcal{U}}-\frac{\mathcal{U}^{2}}{\Delta}\right] \partial_{\mathcal{U}} \Pi=0
$$

(where $\left.\Pi=\oint_{\gamma} \lambda_{5}\right)$. (3.14) gives the Schwarzian differential equation (cf. (G.21)) for $\tau_{5}^{\text {pert }}$

$$
\left\{\tau_{5}^{\text {pert }}, \mathcal{U}\right\}=\frac{2 \mathcal{U}^{2}}{\left(\mathcal{U}^{2}-1\right)^{2}}-\frac{3}{2} \frac{1}{\mathcal{U}^{2}}
$$

(capturing $^{20}$ the perturbative part at large $\mathcal{U}$ by the formal limit $\Lambda \rightarrow 0$ ) with the solution

$$
\tau_{5}^{\text {pert }}=\log \left(\mathcal{U}^{2}-1\right)
$$

\footnotetext{
${ }^{18} \operatorname{Li}\left(e^{\mathcal{A}}\right)$ occurs in $\mathcal{A}_{D}$ as integral (G.23) of $L i_{1}\left(e^{y}\right) \sim \log \left(1-e^{y}\right)$, i.e. $\approx \tau_{5}^{\text {pert }}$ by (3.17), (3.18),(3.19).

${ }^{19}$ Note the formal relation [28] of the 5D massless $N_{f}=1$ curve $y^{2}=\left(x^{2}+s x+1\right)^{2}-c \zeta^{3}(x-1)(c$ a constant which can be gauged by rescalings) with the $4 \mathrm{D}$ massive $N_{f}=1$ curve $\tilde{y}^{2}=\left(\tilde{x}^{2}-u\right)^{2}-c \zeta^{3}(\tilde{x}+m)$ in the quartic form (footn. 27) for $m=\mathcal{U}-1=-\frac{s}{2}-1$ under $\tilde{x}=x+s / 2, u=(s / 2)^{2}-1$.

${ }^{20} \mathrm{cf}$. [28]; note also that using the relations with the four-dimensional quantities this is equivalent to $\partial_{u}\left[4\left(\Lambda^{4}-u^{2}\right) \partial_{u}^{2}-1\right] \Pi=0$ which shows in the bracket the four-dimensional Picard-Fuchs operator
} 
Reinserting the parameter $\zeta$ one gets the $\tau_{5}^{\text {pert }}$ one is actually working with ${ }^{20}$ (cf. below)

$$
\tau_{5}^{\text {pert }}=\log \left(\mathcal{U}^{2}-1\right) \longrightarrow \log \left(\frac{4}{\zeta^{2}}\left(\mathcal{U}^{2}-1\right)\right)
$$

corresponding naturally with $\tau_{4}^{\infty} \sim \log 8 \frac{u}{\Lambda^{2}}$ under (3.7). One has an asymptotic relation

$$
\mathcal{A}=\frac{1}{2} \log \left(\mathcal{U}+\sqrt{\mathcal{U}^{2}-1}\right), \mathcal{U}=\cosh 2 \mathcal{A}, \sqrt{\mathcal{U}^{2}-1}=\sinh 2 \mathcal{A}, \frac{d \mathcal{A}}{d \mathcal{U}}=\frac{1}{2} \frac{1}{\sqrt{\mathcal{U}^{2}-1}}(3.1
$$

(like $u \approx a^{2}$ at infinity in $\left.4 \mathrm{D}\right)$. For the sinh occuring in (3.17) recall that

$$
\log x+\sum_{n \geq 1} \log \left(1+\frac{x^{2}}{n^{2} \pi^{2}}\right)=\log \sinh x=-(\log 2)+x+\log \left(1-e^{-2 x}\right)
$$

This shows correctly the perturbative contribution of the Kaluza-Klein tower

$$
\tau_{5}^{\text {pert }}=\log \sinh ^{2} 2 \mathcal{A}=2 \log 2 \mathcal{A}+2 \sum_{n \geq 1} \log \left(1+\frac{4 \mathcal{A}^{2}}{(n \pi)^{2}}\right)
$$

(because, besides a divergent piece, the terms $\log \left(A^{2}+n^{2} \frac{\pi^{2}}{4 R^{2}}\right)$ occur here). Notice the following two limits of (3.20): first one finds from $\tau_{5}^{\text {pert }}=2 \log \sinh 2 R A=2(2 R A+$ $\left.\log \left(1-e^{-4 R A}\right)-\log 2\right)$ that one has the logarithmic expression $\tau_{5}^{\text {pert }} \rightarrow 2 \log 2 A$ (up to a logarithmically divergent piece in $R$ ) for the 4D limit of $R \rightarrow 0$, as expected from the beta-function in the Seiberg/Witten theory. Secondly, in the 5D decompactification limit $R \rightarrow \infty$, one finds a linear expression from $^{21} \tau_{5, \text { decompact }}^{\text {pert }}=\lim _{R \rightarrow \infty} \frac{\tau_{5}}{\mathbf{2} \pi \mathbf{i} R} \sim 2 \cdot 2 A$.

It will be useful to introduce a further equivalent variable

$$
z=e^{2 \mathcal{A}}=\mathcal{U}+\sqrt{\mathcal{U}^{2}-1}, \mathcal{U}=\frac{z+1 / z}{2}, \sqrt{\mathcal{U}^{2}-1}=\frac{z-1 / z}{2}, \frac{d \mathcal{U}}{\sqrt{\mathcal{U}^{2}-1}}=d \log \gtrsim(3.21)
$$

Actually the more precise relation for $\tau_{5}^{\text {pert }}$ is not (3.16), (3.17) but ${ }^{22}$

$$
\tau_{5}^{\text {pert }}=2 \log \left(1-e^{-4 \mathcal{A}}\right)=\log 4 \sinh ^{2} 2 \mathcal{A}-4 \mathcal{A}=\log 4 \frac{\mathcal{U}^{2}-1}{z^{2}} \longrightarrow \log \frac{4}{\zeta^{2}} \frac{\mathcal{U}^{2}-1}{z^{2}}
$$

One gets from (3.10), (3.22) for the dual period in the weak coupling limit of large $U$

$$
\begin{aligned}
\mathcal{A}_{D} & =\frac{1}{2} \int \log \left(\frac{4}{\zeta^{2}} \frac{\mathcal{U}^{2}-1}{z^{2}}\right) \frac{d \mathcal{U}}{\sqrt{\mathcal{U}^{2}-1}}=\frac{1}{2}\left(\int \log \frac{4}{\zeta^{2}} d \log z+\int \log \left(\frac{z-\frac{1}{z}}{2 z}\right)^{2} d \log z\right) \\
& =\frac{1}{2}\left(\operatorname{Li}\left(1 / z^{2}\right)+\log \zeta \log \left(1 / z^{2}\right)\right)=\frac{1}{2}\left(\operatorname{Li}\left(e^{-4 \mathcal{A}}\right)-(\log \zeta) 4 \mathcal{A}\right)
\end{aligned}
$$

So one gets ${ }^{22}$ for the monodromy around $\mathcal{U} \approx \infty$

$$
\left(\begin{array}{c}
\mathcal{A}_{D} \\
\mathcal{A} \\
1
\end{array}\right)=\left(\begin{array}{c}
\frac{1}{2} \operatorname{Li}(x)+\frac{1}{2} \log \zeta \log (x) \\
-\frac{1}{4} \log x \\
1
\end{array}\right), \quad M_{\infty}=\left(\begin{array}{ccc}
1 & 8 \pi i & 2 \pi i \log \zeta \\
0 & 1 & -\pi i \\
0 & 0 & 1
\end{array}\right)
$$

\footnotetext{
${ }^{21}$ rescaling $\tau_{5}$ so that it corresponds to the gauge coupling $\tau_{5}=1 / g_{5}^{2}$ of the $5 \mathrm{D}$ theory; $\tau_{5}^{\text {pert }}$ and $\tau_{f}$ in (3.20) and (3.2) are in a sense $4 \mathrm{D}$ as $\mathbf{S}^{\mathbf{1}}$ is compact, (3.4) is 5D; cf. the discussion after (3.2)

${ }^{22}$ cf. [28]; for a corresponding analysis based on (3.17) cf. app. C (cf. also remark after (3.2))
} 


\section{Discussion}

Although it may be already clear from the foregoing presentation let us point to the direction in which we see the significance of the mentioned relations. We compared a number of scenarios where the dilogarithm, respectively structures associated to it, occured as a quantum correction. Given the markedly special functional structure of the dilogarithm ${ }^{23}$ (nobody would intend to ask for an underlying pattern of different occurrences of the sine function, for example) we think these similarities are worth to be remarked in the first instance and worth to be asked for being understood by some explanation. The proper reason of the fact that juxtapositions such as the ones above are possible should be that the occurrence of the dilogarithm (or variants of it) as a quantum correction in the examples mentioned has a common origin: this may happen either by direct connections between the respective scenarios or by some more general principle (like for the logarithm in one-loop corrections).

For this reason we give a certain weight to the reformulation of the transformation properties of the dilogarithm via the upper triangular (Heisenberg) group. It is via this device that we try to suggest a connection to other quantum corrections. A well-known case concerns the Seiberg/Witten non-perturbative solution of $4 \mathrm{D} N=2 S U(2)$ gauge theory where logarithmic terms near special points of the moduli space are captured in the monodromy description by upper triangular (integral) $2 \times 2$ matrices. When enhanced with a further (mass) parameter this description extends to similar $3 \times 3$ matrices, as we recall in (D.10); the precise form (D.14) does not give the proper dilog Heisenberg monodromy. By contrast, when one chooses for the additional mass scale the KK-scale $m=1 / R_{5}$ of $5 \mathrm{D}$ Seiberg/Witten theory compactified on a circle of radius $R_{5}$, one gets indeed the correct monodromy for the dilogarithm up to a specific linear modification, as described in sect. 3 (we recall necessary background on the relevant associated Schwarzian differential equation and the corresponding Seiberg/Witten theory in app.'s G and D); concerning the modification cf. also [8], sect 4.4. We propose that in this case the reason lying behind this occurrence of the dilogarithm is the interpretation of the dual period $a_{D}$ (resp. rather its five-dimensional incarnation) as a conifold period after a stringy embedding of the gauge theory (the necessary period relations are recalled in app. E). So our reference scenario for the meaning of the dilogarithm is the conifold (and the associated superpotential), cf. [31] in type IIA (resp. for the $M$-theory case [8]).

\footnotetext{
${ }^{23}$ Note that we are not concerned with the expansion of expressions (like prepotentials in $N=2$ theories) in polylog's but rather with the much more specific case of the pure dilog.
} 
Further, given the reformulation via the Heisenberg group, another possible relation comes to mind: besides the universal scenario given by considering (not an arbitrary 3cycle in type IIA on a Calabi-Yau but) the local conifold limit there is also the type IIA universal hypermultiplet. For the conifold we think in the technical description in terms of the $M$-theory lift and the corresponding dilogarithm, cf. [8]; this has the advantage of changing the two sides of the transition to two symmetrical $\mathbf{S}^{\mathbf{3}}$ 's. The universal hypermultiplet framework has then firstly a similar exchange symmetry in (2.13) resp. the corresponding conjugation exchange in the 12 and 23 positions in (2.16). Further these two universal structures compare via the transformation behaviour (2.14), resp. (2.16), and (2.2) and (2.3); further via the real invariant functions (2.15) and (2.5); finally in light of the discussion after (2.20). It seems that the two real degrees of freedom embodied in $C \sim c \epsilon_{i j k}$ (resp. for the conjugate polarization $\epsilon_{\bar{i} \bar{j} \bar{k}}$ ) in the universal hypermultiplet case correspond in some sense with the dual moduli related to the $\mathbf{S}^{\mathbf{3}}$ 's in the $M$-theory lift of the conifold; it should be interesting to understand this further.

Yet another indication of such a connection comes from a different perspective (cf. remark 1 of section 2). After extracting an appropriate power (conceptually justifiable [13], cf. footn. 9) of the metric corrections in the hyperkähler limit [2] one ends up with a correspondingly twisted version of the dilogarithm; as discussed after (2.28), such a metric term fits nicely with the $2 \operatorname{Re} S d c d \bar{c}$ in (2.17) and the proposed correspondences for the quantities involved. These observations point into the same direction as above. ${ }^{24}$

Clearly the most interesting question would be whether the occurrence of the dilogarithm (or the Heisenberg group shifts) in different scenarios is universal as a consequence of certain physical principles, like in logarithmic one-loop corrections of gauge couplings; the theta-angle shifts responsible for that behaviour (described by integral upper triangular $2 \times 2$ matrices) may be now replaced with the more complicated shifts (described by corresponding $3 \times 3$ matrices) related to two 'dual' objects like $\Omega_{i j k}$ and $\bar{\Omega}_{\bar{i} \bar{j} \bar{k}}$ or $\mathbf{S}_{\mathbf{Q}}^{\mathbf{3}}$ and $\mathbf{S}_{\mathbf{D}}^{\mathbf{3}}$ (respectively the associated $c, \bar{c}$ or the corresponding $M$-theory moduli).

If the way we presented things to make them amenable to a useful comparison would have convinced the reader that such an underlying principle might be reasonable to ask for and interesting to formulate this paper would have fulfilled its task.

\footnotetext{
${ }^{24}$ Another parallelism of occurrences of the dilogarithm in differing situations was noticed when the classical $(N=0)$ Schwinger computation was used [30] to derive corrections in $(N=1,2)$ supersymmetric theories (cf. Introduction, footn. 10; for associated background cf. app. F). Besides this known relation an instanton reformulation [12] of the classical Schwinger computation is formally even more closely related (remark 3, sect. 2) to an (instanton) derivation of the dilogarithm.
} 


\section{Appendix}

\section{A The cone on $\mathrm{S}^{3} \times \mathrm{S}^{3}$}

The cone on $\mathbf{S}^{\mathbf{3}} \times \mathbf{S}^{\mathbf{3}}$ has the $G_{2}$ holonomy metric $\left(r \geq r_{0}\right)$ [1], [8]

$$
d s^{2}=\frac{d r^{2}}{1-\left(\frac{r_{0}}{r}\right)^{3}}+\frac{r^{2}}{36}\left(d a^{2}+d b^{2}+d c^{2}-\left(\frac{r_{0}}{r}\right)^{3}\left(d a^{2}-\frac{1}{2} d b^{2}+d c^{2}\right)\right)
$$

Concerning metric perturbations which preserve $G_{2}$ holonomy one finds (up to terms $y^{2} \mathcal{O}\left(\left(\frac{r_{0}}{y}\right)^{6}\right)$ in a new radial coordinate $\left.y\right)$ with $\left(f_{1}, f_{2}, f_{3}\right)=(1,-2,1)$

$$
d s^{2}=d y^{2}+\frac{y^{2}}{36}\left(d a^{2}+d b^{2}+d c^{2}-\frac{1}{2}\left(\frac{r_{0}}{y}\right)^{3}\left(f_{1} d a^{2}+f_{2} d b^{2}+f_{3} d c^{2}\right)\right)
$$

At small $r_{0}$ or large $y$ one finds the conical metric with the full $\Sigma_{3}$ symmetry. One has for the volume of $Q_{i}$ and the $y$-dependent volume of $D_{i}$ embedded in $X_{i}$ (at large $y$ )

$$
\begin{aligned}
\operatorname{vol} Q_{i} & =2 \pi^{2} r_{o}^{3} \\
\operatorname{vol} D_{i} & =\frac{2 \pi^{2}}{27} y^{3}\left(1+\frac{3}{8} f_{i}\left(\frac{r_{o}}{y}\right)^{3}+\mathcal{O}\left(\left(\frac{r_{0}}{y}\right)^{6}\right)\right) \approx \log c+\frac{1}{72} f_{i} \operatorname{vol} Q_{i}
\end{aligned}
$$

Here, the first correction to the divergent piece is the finite volume defect $\frac{1}{72} f_{i} \operatorname{vol}\left(\mathbf{S}_{\mathbf{r}_{\mathbf{0}}}^{\mathbf{3}}\right)$.

A holomorphic observable on $\mathcal{N}$ must combine as SUSY partners the $C$-field period $\alpha_{i}=\int_{D_{i}} C$ with an order $1 / r^{3}$ metric perturbation (w.r.t. the conical metric), as in

$$
y_{i}=\exp \left(k f_{i}+i\left(\alpha_{i+1}-\alpha_{i-1}\right)\right)
$$

(with $\left.\prod_{i} y_{i}=1\right)$. Actually one works with the quantity $\left(\right.$ so $\left.\eta_{i}=\left(y_{i-1}^{2} y_{i}\right)^{1 / 3}, y_{i}=\frac{\eta_{i+1}}{\eta_{i-1}}\right)$

$$
\eta_{i}=\exp \left(\frac{k}{3}\left(f_{i-1}-f_{i+1}\right)+i \alpha_{i}\right)
$$

$\eta_{i}$ is $0,1, \infty$ at $P_{i-1}, P_{i}, P_{i+1}$ where $P_{i}$ is the semiclassical end $\operatorname{vol}\left(Q_{i}\right) \approx \infty$. One also has the membrane instanton amplitude as local coordinate $u_{i}$ at $P_{i}$ (vanishing there)

$$
u_{i}=\exp \left(-T \operatorname{vol}\left(Q_{i}\right)+i \int_{Q_{i}} C\right)
$$

We denote by $\Phi_{i}$ the physical modulus to which $u_{i}$ is related via $u_{j}=e^{i \Phi_{j}}\left(Q_{i}\right.$ is a supersymmetric cycle so $\left.\Upsilon\right|_{Q_{i}}$ is the volume form), i.e. $\Phi_{j}=\int_{Q_{j}} C+i \Upsilon=\phi_{j}+i \operatorname{vol}\left(Q_{j}\right)$.

In a semiclassical regime with $D_{i}=0$ one has $Q_{i}=\mp D_{i+1}$ so that $\mp \int_{D_{1}} C=\int_{Q_{3}} C$, what is (with holomorphy) tantamount to having $\eta_{i+1} \sim u_{i}$ to first order. We assume that such a relation persists ${ }^{25}$ so that

$$
\beta u_{i}=\eta_{i}
$$

${ }^{25}$ Thereby a relation $\eta_{i-1}=\frac{1}{1-\eta_{i}}$ holds also for the $u_{i}: u_{3}=\beta u_{1}=\frac{1}{1-u_{1}}$ 


\section{B The universal hypermultiplet moduli space}

One may parametrize the coset space $S U(2,1) / U(1)$ by two complex scalars $z_{1}$ and $z_{2}$ in the open four-ball $\left|z_{1}\right|^{2}+\left|z_{2}\right|^{2}<1$ with Kähler potential

$$
K=-\log \left(1-\left|z_{1}\right|^{2}-\left|z_{2}\right|^{2}\right)
$$

and the Bergman metric with a left $S U(2,1)$ isometry group

$$
d s^{2}=\frac{d z_{1} d \bar{z}_{1}+d z_{2} d \bar{z}_{2}}{1-z_{1} \bar{z}_{1}-z_{2} \bar{z}_{2}}+\frac{\left(\bar{z}_{1} d z_{1}+\bar{z}_{2} d z_{2}\right)\left(z_{1} d \bar{z}_{1}+z_{2} d \bar{z}_{2}\right)}{\left(1-z_{1} \bar{z}_{1}-z_{2} \bar{z}_{2}\right)^{2}}
$$

One may also switch to polar coordinates $(r<1, \theta \in[0, \pi), \phi \in[0,2 \pi), \psi \in[0,4 \pi))$

$$
z_{1}=r \cos \frac{\theta}{2} e^{i \frac{\psi+\phi}{2}} \quad, \quad z_{2}=r \sin \frac{\theta}{2} e^{i \frac{\psi-\phi}{2}}
$$

which gives with the $S U(2)$ one-forms $\left(d \sigma_{i}=-\frac{1}{2} \epsilon_{i j k} \sigma_{j} \wedge \sigma_{k}\right)$

$$
\begin{aligned}
\sigma_{1} & =\cos \psi d \theta+\sin \psi \sin \theta d \phi \\
\sigma_{2} & =-\sin \psi d \theta+\cos \psi \sin \theta d \phi \\
\sigma_{3} & =d \psi+\cos \theta d \phi
\end{aligned}
$$

and the complex vielbeins

$$
\begin{gathered}
u=e^{2}+i e^{1} \quad, \quad v=e^{r}+i e^{3} \\
e^{r}=\frac{d r}{\left(1-r^{2}\right)} \quad, \quad e^{1 / 2}=\frac{r}{2 \sqrt{1-r^{2}}} \sigma_{1 / 2} \quad, \quad e^{3}=\frac{r}{2\left(1-r^{2}\right)} \sigma_{3}
\end{gathered}
$$

for the Kähler metric

$$
\begin{aligned}
d s^{2} & =\frac{d r^{2}}{\left(1-r^{2}\right)^{2}}+\frac{r^{2}}{4\left(1-r^{2}\right)}\left(\sigma_{1}^{2}+\sigma_{2}^{2}\right)+\frac{r^{2}}{4\left(1-r^{2}\right)^{2}}\left(\sigma_{3}^{2}\right) \\
& =u \bar{u}+v \bar{v}=\left(e^{r}\right)^{2}+\left(e^{1}\right)^{2}+\left(e^{2}\right)^{2}+\left(e^{3}\right)^{2}
\end{aligned}
$$

The relation to the variables $S$ and $c$ is given by

$$
z_{1}=\frac{1-S}{1+S} \quad, \quad z_{2}=\frac{2 c}{1+S} \quad S=\frac{1-z_{1}}{1+z_{1}} \quad, \quad c=\frac{z_{2}}{1+z_{1}}
$$

and makes, via a Kähler transformation, (B.1) equivalent to their Kähler potential

$$
K=-\log (S+\bar{S}-2 c \bar{c})
$$

The toroidal fibration (B.3) resp. (2.21) is analogous to the fibration one gets in the case of signature $S U(3) / U(2) \cong \mathbf{P}_{\mathbf{C}}^{\mathbf{2}}$ (where $e^{-K}=1+r^{2}$ instead of (B.1)) [25]. 


\section{The alternative evaluation in 5D gauge theory}

There is an ambiguity whether to carry along the linear piece $\mathcal{A}$ in $\tau$ in (3.22) (cf. remark after (3.2)). Starting instead of (3.22) from (3.17) one gets

$$
\begin{aligned}
\mathcal{A}_{D} & =\int \tau_{5}^{\text {pert }} d \mathcal{A}=\frac{1}{2} \int \log \left(\frac{4}{\zeta^{2}}\left(\mathcal{U}^{2}-1\right)\right) \frac{d \mathcal{U}}{\sqrt{\mathcal{U}^{2}-1}} \\
& =\frac{1}{2}\left(\operatorname{Li}\left(1 / z^{2}\right)+\log \zeta \log \left(1 / z^{2}\right)+\log ^{2} z\right) \\
& =\frac{1}{2}\left(\operatorname{Li}\left(e^{-4 \mathcal{A}}\right)-4(\log \zeta) \mathcal{A}+4 \mathcal{A}^{2}\right)
\end{aligned}
$$

Note that in contrast to (3.23) here a quadratic term in $\mathcal{A}$ appears in $\mathcal{A}_{D}$. Now for $-4 \mathcal{A}=i \phi \in i \mathbf{R}$ being purely imaginary (think of this special case like having a coupling constant $\theta+i \frac{4 \pi}{g^{2}}$ of $\theta=0$ ) one obtains with (G.24) that this quadratic term vanishes

$$
\mathcal{A}_{D}=\frac{1}{2}\left(-\left(\frac{\pi i}{2}+\log \zeta\right) 4 \mathcal{A}+\frac{\pi^{2}}{6}+i \operatorname{Im} L i\left(e^{-4 \mathcal{A}}\right)\right)
$$

To understand this occurrence of $\operatorname{Im} L i\left(e^{-4 \mathcal{A}}\right)=I(\phi)$ in $\mathcal{A}_{D}$ more directly compare (starting from $\tau_{5}^{\text {pert }}=2 \log \frac{2}{\zeta}+2 \log \left(-i \sin \frac{\phi}{2}\right)$ )

$$
\begin{aligned}
I(\phi) & =-\int \log 2 \sin \frac{\phi}{2} d \phi=-\left((\log 2) \phi+\int \log \sin \frac{\phi}{2} d \phi\right) \\
\mathcal{A}_{D} & =\int \tau_{5}^{\text {pert }} d \mathcal{A}=-\frac{i}{2}\left(\log \frac{2}{\zeta}\right) \phi-\frac{i}{2} \int \log \left(-i \sin \frac{\phi}{2}\right) d \phi \\
& =-\frac{i}{2}\left(\left(\log \frac{2}{\zeta}-\frac{i \pi}{2}\right) \phi+\int \log \sin \frac{\phi}{2} d \phi\right)
\end{aligned}
$$

where the last line of (C.3) gives back (C.2) up to an integration constant. Now

$$
\begin{aligned}
\left(\begin{array}{c}
2 \mathcal{A}_{D} \\
-4 \mathcal{A} \\
1
\end{array}\right) & =\left(\begin{array}{c}
L i(y)+\log \zeta \log y+\frac{1}{4} \log ^{2} y \\
\log y \\
1
\end{array}\right) \\
M_{\infty} & =\left(\begin{array}{ccc}
1 & -2 \pi i & (2 \log \zeta+2 \pi i) 2 \pi i \\
0 & 1 & 2 \cdot 2 \pi i \\
0 & 0 & 1
\end{array}\right)
\end{aligned}
$$

(the quadratic term $\mathcal{A}^{2}$ of (C.1) caused by the linear piece in $\tau$ can be seen also in (C.4), (C.5)). The relations $\tau_{5}^{\text {pert }}{ }^{\infty}=2 \log \left(\frac{2}{\zeta} \mathcal{U}\right)$ and $\mathcal{A}^{\infty}=\frac{1}{2} \log 2 \mathcal{U}$ give directly the asymptotic relation $\mathcal{A}_{D}^{\infty} \approx \frac{1}{2}\left(-4 \mathcal{A}^{\infty} \log \zeta+4 \mathcal{A}^{\infty 2}\right)$, which is the limit case of (C.1). 


\section{D $\quad 4 \mathrm{D} N=2 S U(2)$ field theory}

Four-dimensional $N=2$ supersymmetric pure $S U(2)$ gauge theory has as Seiberg/Witten curve [19] the elliptic curve $E_{u}$ (varying over the $u$-plane) given by

$$
y^{2}=\left(x^{2}-\Lambda^{4}\right)(x-u)
$$

The meromorphic one-form (with the constant $c=\frac{\sqrt{2}}{4 \pi}$ )

$$
\lambda_{4}=c(x-u) \frac{d x}{y}
$$

leads to the periods ( $\alpha, \beta$ the one-cycles of $E_{u}$ over $\left[-\Lambda^{2}, \Lambda^{2}\right]$ and $\left[\Lambda^{2}, u\right]$, respectively)

$$
a=\oint_{\alpha} \lambda_{4}, a_{D}=\oint_{\beta} \lambda_{4}
$$

where the dual parameter $a_{D}$ is the derivative of the prepotential $\mathcal{F}$

$$
a_{D}=\partial \mathcal{F} / \partial a
$$

From $a$ and $a_{D}$ one gets the expression for the $N=1$ Kähler potential

$$
K=-\operatorname{Im} a \bar{a}_{D}
$$

Further one has the relation [21] (for second equality [23] cf. (D.16) and sect. E, app.)

$$
\frac{1}{2 \pi i} u=\frac{1}{4}\left(2 \mathcal{F}-a \partial_{a} \mathcal{F}\right)=-\partial_{S} \mathcal{F}
$$

When expressed in the rescaled dimensionless variable $\tilde{u}:=\frac{u}{\Lambda^{2}}$ the three special points are $\tilde{u}= \pm 1, \infty$. As the Legendre function $\lambda$ (cf. (G.11)) is gauged on the three special points $0,1, \infty$ let us switch the gauges $(+1,-1, \infty) \longrightarrow(0,1, \infty)$ which is effected by $z \longrightarrow \underline{z}:=\frac{1-z}{2}$. One has then

$$
\underline{\tilde{u}}(\tau):=\frac{1-\tilde{u}(\tau)}{2}=\lambda(\tau)
$$

Note that one has a certain (formal) triality symmetry operating on the quantum moduli space as one finds, with the identification (D.7), that $\mathbf{P}_{\underline{\underline{u}}}^{1}=\Gamma(2) \backslash \mathbf{H}_{\mathbf{2}}$ has an $\Sigma_{3}$ action with quotient $\Sigma_{3} \backslash \mathbf{P}_{\lambda(\tau)}^{1}=\mathbf{P}_{\mathbf{j}(\tau)}^{1}$ where $j=\frac{27}{4} \prod_{i \in \mathbf{Z}_{3}}\left(\beta^{i} \lambda-\beta^{i+1} \lambda\right.$ ) (cf. (G.14)). Clearly in the actual physical interpretation this $\Sigma_{3}$ symmetry is broken down to a $\mathbf{Z}_{\mathbf{2}}$ symmetry between $\tilde{u}=+1$ and $\tilde{u}=-1$. 
One has further that $\frac{\partial}{\partial u} \lambda_{4}$ is equal to $-\frac{c}{2} \frac{d x}{y}$, i.e. to the holomorphic one-form. The $u$-derivatives of $a$ and $a_{D}$ give $^{26}$ thus the (holomorphic) periods $b_{1}$ and $b_{2}$ of the elliptic curve (cf. [28]; here $F(z)=F_{\frac{1}{2} \frac{1}{2} 1}(z)$ the hypergeometric function, cf. sect. G)

$$
\frac{1}{\Lambda} \frac{d a}{d \tilde{u}}=\frac{1}{2} \sqrt{\beta \underline{\tilde{u}}} F(\beta \underline{\tilde{u}}) \quad, \quad \frac{1}{\Lambda} \frac{d a_{D}}{d \tilde{u}}=\frac{i}{2} F(\underline{\tilde{u}})
$$

which fulfil the Picard-Fuchs equation

$$
\left(1-\tilde{u}^{2}\right) \partial_{\tilde{u}}^{2} \Pi-\frac{1}{4} \Pi=0
$$

\section{Inclusion of matter}

For the sake of comparison with results in the five-dimensional theory let us consider also the case with matter. First note that quite generally the monodromy has the following block-upper-triangular form

$$
\left(\begin{array}{c}
a_{D} \\
a \\
1
\end{array}\right) \longrightarrow\left(\begin{array}{cc}
N & v \\
0 & 1
\end{array}\right)\left(\begin{array}{c}
a_{D} \\
a \\
1
\end{array}\right)
$$

where $N \in S l(2, \mathbf{Z})$ and $v$ is an integral column vector. We recall how this possibility (not realised in the pure gauge theory) is actually realised in the theory with matter.

Recall first the central charge and mass of a BPS particle in the pure gauge theory

$$
M=\sqrt{2}|Z| \quad, \quad Z=n_{e} a+n_{m} a_{D}
$$

By contrast, let us consider the case $N_{f}=1$ with one additional hypermultiplet as matter; this consists of two chiral $N=1$ multiplets $Q, \tilde{Q}$ with $Q$ a quark in the fundamental representation (for $S U(2) \tilde{Q}$ will be again in the fundamental representation). The global symmetry on the $u$-plane (we are discussing the Coulomb branch) changes from $\mathbf{Z}_{\mathbf{2}}$ to $\mathbf{Z}_{\mathbf{3}}$ (coming from the anomaly free subgroup $\mathbf{Z}_{\mathbf{1 2}}$ of $U(1)_{R}$ ) and there are three singularities related by this symmetry. Furthermore the instanton terms go in powers of $\Lambda_{1}^{3}$ (instead of $\Lambda^{4}$ ) and terms with an odd number of instantons vanish because of an anomalous "parity" $\mathbf{Z}_{\mathbf{2}}$ in $O\left(2 N_{f}\right)$ sending the quark $Q$ to the antiquark $\tilde{Q}$. The curve is then $y^{2}=x^{2}(x-u)+t \Lambda_{1}^{6}\left(\right.$ with $t$ a constant which can be absorbed in $\left.\Lambda_{1}\right)$.

Now let us turn on an $N=2$ invariant mass term for the matter. The curve is modified to $y^{2}=x^{2}(x-u)+\Lambda_{1}^{3}\left(\frac{1}{4} m x+t \Lambda_{1}^{3}\right)$ where $t=-\frac{1}{64}$ (for large $m$, with $\Lambda_{0}^{4}=m \Lambda_{1}^{3}$

\footnotetext{
${ }^{26}$ Note that the normalisations used ammount to $\tau\left(E_{u}\right)=\frac{d a_{D}}{d a}=i \frac{F\left(\alpha \beta^{2} \tilde{\tilde{u}}\right)}{F(\beta \underline{\tilde{u}})}=\tau(\beta \underline{\tilde{u}})$ with $(\mathrm{G} .20)$ and (G.19); cf. now footn. 38 .
} 
held fixed, this reduces to the curve ${ }^{27}$ of the pure gauge theory). The elementary particles (like electrons or quarks) would not have $m_{e}=\sqrt{2}|a|$ but $m_{e}=\sqrt{2}\left|a+\frac{m}{\sqrt{2}}\right|$; so when additional abelian conserved charges are present they contribute to the central charge and this modifies (D.11) by the occurrence of the $U(1)$ charge $S$ of the hypermultiplet to

$$
Z=n_{e} a+n_{m} a_{D}+S \frac{m}{\sqrt{2}}
$$

At $a_{ \pm}= \pm \frac{m}{\sqrt{2}}$ one of the elementary quarks becomes massless. The behaviour at $a_{+}=a_{0}$

$$
\begin{aligned}
a & \approx a_{0} \\
a_{D} & \approx c-\frac{i}{2 \pi}\left(a-a_{0}\right) \log \left(a-a_{0}\right)
\end{aligned}
$$

(with $c$ a constant) implies for the monodromy around $a_{0}$

$$
\left(\begin{array}{c}
a_{D} \\
a \\
\frac{m}{\sqrt{2}}
\end{array}\right) \longrightarrow\left(\begin{array}{ccc}
1 & 1 & -1 \\
0 & 1 & 0 \\
0 & 0 & 1
\end{array}\right)\left(\begin{array}{c}
a_{D} \\
a \\
\frac{m}{\sqrt{2}}
\end{array}\right)
$$

Stringy realization of the $N=2 \longrightarrow N=1$ mass breaking

The quantum corrected version $W=m u$ of the classical $\left(u=<\operatorname{tr} \Phi^{2}>\approx a^{2} / 2\right)$ mass deformation in the field theory (providing mass to the scalar partner $\Phi$ of the vector multiplet which leads to the breaking $N=2 \longrightarrow N=1$ ) is realised [4] as a flux induced superpotential $W=\int_{W} \Omega \wedge H_{3}$ in the type IIB string, essentially because $u$ occurs according to (D.6), (E.9) among the Calabi-Yau periods. As near $\tilde{u}= \pm 1$ a monopole and a dyon become massless one gets by including the light states

$$
W=m u+\left(a_{D}-a_{0}\right) \phi \tilde{\phi}
$$

which leads by minimisation to monopole condensation $\phi \tilde{\phi}$ and locking on the monopole point $\tilde{u}= \pm 1 \longleftrightarrow a_{D}=a_{0}$. The stringy realization proposed in [4] of this scenario started from the type IIA superpotential (cf. sect. E)

$$
W_{\text {flux }} \sim \int_{X} H_{2} \wedge t \wedge t \sim\left(\int_{\mathbf{P}_{\mathbf{b}}^{\mathbf{1}}} H_{2}\right) \cdot \operatorname{vol}(K 3)=n_{\text {flux }} \partial_{s} \mathcal{F}
$$

where the type IIA periods $S$ and $\partial \mathcal{F} / \partial S$ correspond to $\operatorname{vol}\left(\mathbf{P}_{\mathbf{b}}^{\mathbf{1}}\right)$ and $\operatorname{vol}(K 3) .{ }^{28}$

\footnotetext{
${ }^{27}$ Note that in the normalizations used in the pure gauge theory [19] one works with the $\Gamma(2)$ curve $y^{2}=\left(x^{2}-\Lambda^{4}\right)(x-u)$ whereas in the framework of general $N_{f}[20]$ one works, even for $N_{f}=0$, with the $\Gamma_{0}(4)$ curve $y^{2}=x^{2}(x-u)+\frac{1}{4} \Lambda^{4} x$. Note further that besides these cubic forms of the representation of the elliptic curve there are also corresponding quartic forms in use $\left(\Gamma^{0}(4)\right.$ curves; cf. for example [22]), namely $y^{2}=\left(x^{2}-u\right)^{2}-\Lambda_{0}^{4}$ and $y^{2}=\left(x^{2}-u\right)^{2}-\Lambda_{1}^{3}(x+m)$ for $N_{f}=0$ and 1 , respectively.

${ }^{28}$ Due to analytic continuation (E.10) this has to be refined [24]: actually $W=m u \sim 2 i \Xi_{\infty}^{2}+\Xi_{\infty}^{4}=$ $2 i t+\partial_{s} \mathcal{F}$.
} 


\section{E $\quad 4 \mathrm{D} N=2 S U(2)$ field theory from IIA-theory}

Let us reconsider the gauge theoretic descriptions from the perspective of an embedding into the type IIA string on a Calabi-Yau manifold. There one has for the prepotential ( $k$ counting the multiple covers)

$$
\mathcal{F}=\frac{1}{6} \sum C_{i j k} T_{i} T_{j} T_{k}+\text { lowerterms }+\sum_{\left(d_{i}\right) \geq 0, k \geq 1} \frac{c_{\left(d_{i}\right)}}{k^{3}} \prod_{i} e^{-k d_{i} T_{i}}
$$

with the $\left(d_{i}\right)$ indicating the degree of the primitive world-sheet instantons.

\section{A local Calabi-Yau model}

To become more specific let us consider a local model for a $S U(2)$ singularity, i.e. an $A_{1}$ fibration. This is given by a CY elliptically fibered over one of the Hirzebruch surfaces $F_{0}, F_{1}, F_{2}$; we will consider the case of $F_{0}=\mathbf{P}_{\mathbf{b}}^{1} \times \mathbf{P}_{\mathbf{f}}^{1}$ in the limit of making the size of the fibre large, i.e. we look only at the neighborhood of the $F_{0}$ inside the CY which is then the local CY isomorphic to the total space of the normal bundle of $F_{0}$ (inside the original CY) given by the line bundle $\mathcal{O}(-2) \times \mathcal{O}(-2)$ over $F_{0}$.

We are interested in the $U(1)$ related to $T_{f}$ so let us note that

$$
\frac{1}{g_{Y M}^{2}} \sim T_{b} \quad, \quad m_{W}=T_{f} \cdot \frac{1}{g_{s} l_{s}}
$$

as the $W$-boson will be represented by a $\mathrm{D} 2$-brane wrapped on $\mathbf{P}_{\mathbf{f}}^{1}$. The relevant limits are then the weak couling limit $T_{b} \rightarrow \infty$ and the singular limit $T_{f} \rightarrow 0$ leading to enhanced gauge symmetry. The gauge coupling for the $U(1)$ related to $T_{f}$ is given by

$$
\tau_{f}=i \frac{\partial^{2} \mathcal{F}}{\partial T_{f}^{2}}=i \sum_{n, m \geq 0 ; k \geq 1} m^{2} \frac{c_{n, m}}{k} q_{b}^{k n} q_{f}^{k m}
$$

$\left(q_{i}=e^{-T_{i}}\right)$. The scaling limit [18] (which allows to extract the field-theoretic quantities)

$$
T_{f} \sim \epsilon \quad, \quad T_{b} \sim \log \frac{1}{\epsilon^{4}}
$$

gives, assuming $c_{n, m} \sim \gamma_{n} m^{4 n-3}$ ( $m$ large, $n$ fixed), for the leading ( $\epsilon$-independent) term the sum of space-time instanton corrections of a gauge theory prepotential

$$
\tau_{f} \sim \sum_{n} \gamma_{n}\left(\frac{e^{-T_{b}}}{T_{f}^{4}}\right)^{n}
$$

For the scales note $\Lambda_{Q C D}^{4}=\Lambda_{U V}^{4} e^{-\frac{1}{g_{U V}^{2}}}$ where $\Lambda_{U V} \approx \frac{1}{g_{s}} m_{s}=\frac{1}{g_{s} l_{s}}$ : the expansion parameter in (E.5) is $\frac{e^{-T_{b}}}{T_{f}^{4}}=\frac{e^{-T_{b}}}{\left(g_{s} l_{s} M_{W}\right)^{4}}$ by (E.2), i.e. $\frac{\Lambda_{Q C D}^{4}}{M_{W}^{4}}$ for the asserted $\Lambda_{U V}=\frac{1}{g_{s} l_{s}}$

$$
T_{f}=g_{s} l_{s} M_{W} \quad, \quad e^{-T_{b}}=\left(g_{s} l_{s}\right)^{4} \Lambda_{Q C D}^{4}
$$




\section{A global Calabi-Yau model}

To make closer contact with the field theory it is useful to go to a special global Calabi-Yau model, in our case of $S U(2)$ gauge theory to the $S T$-Calabi-Yau $X=X_{S T}$ given by the hypersurface $\mathbf{P}_{11226}(12)$. It is $K 3$-fibered over a $\mathbf{P}^{\mathbf{1}}$ base and its two Kähler classes $S$ and $T$ measure the complex volume of the base and the fibre, respectively. One uses an integral symplectic basis for the periods in type IIB of its mirror Calabi-Yau $W$, or equivalently a basis for $H_{3}(W, \mathbf{Z})$ at the point of maximal unipotent monodromy (corresponding in $X$ to the large volume limit). At this point $z^{A}=0$ one has a unique analytic period, normalized as $X^{0}=1+\mathcal{O}(z)$, and $m=h^{2,1}(W)$ logarithmic periods $X^{A}$ which provide natural special complex Kähler coordinates $t^{A}=\frac{X^{A}}{X^{0}}=\frac{1}{2 \pi i} \log z^{A}+\mathcal{O}(z)$. The prepotential is homogeneous of degree two in the periods $X^{I}$ (with $q^{A}=e^{2 \pi i t^{A}}$ )

$$
\begin{aligned}
F & =-\frac{C_{A B C}}{3 !} \frac{X^{A} X^{B} X^{C}}{X^{0}}+\frac{n_{A B}}{2} X^{A} X^{B}+c_{A} X^{A} X^{0}-i \chi \frac{\zeta(3)}{2(2 \pi)^{3}}\left(X^{0}\right)^{2}+\left(X^{0}\right)^{2} f(q) \\
& =\left(X^{0}\right)^{2} \mathcal{F}=\left(X^{0}\right)^{2}\left(-\frac{C_{A B C}}{3 !} t^{A} t^{B} t^{C}+\frac{n_{A B}}{2} t^{A} t^{B}+c_{A} t^{A}-i \chi \frac{\zeta(3)}{2(2 \pi)^{3}}+f(q)\right)(\text { E. } 7)
\end{aligned}
$$

The $C_{A B C}=\int_{X} J_{A} J_{B} J_{C}$ are the classical intersection numbers in the type IIA interpretation $\left(J_{A}\right.$ integral $(1,1)$ forms spanning the Kähler cone), and $c_{A}=\frac{1}{24} \int_{X} c_{2} J_{A}$; the world sheet instanton expansion in $q$ is determined via mirror symmetry using type IIB on $W$.

This defines an integral basis for the periods

$$
\Xi_{\infty}=\left(\begin{array}{c}
X^{0} \\
X^{A} \\
\frac{\partial F}{\partial X^{A}} \\
\frac{\partial F}{\partial X^{0}}
\end{array}\right)=X^{0}\left(\begin{array}{c}
1 \\
t^{A} \\
\frac{\partial \mathcal{F}}{\partial t^{A}} \\
2 \mathcal{F}-t^{A} \frac{\partial F}{\partial t^{A}}
\end{array}\right)
$$

One has the leading order relations to the large complex structure variables $z_{t} \propto$ $e^{-T}, z_{s} \propto e^{-S}$ (we use also the common rescaled variables $x=1728 z_{t}, y=4 z_{s}$ ). To make contact with the field theory one first brings the relevant divisors in the two-dimensional moduli space to generic intersections. The order two tangency at $p=(x, y)=(1,0)$ between the conifold divisor $\Delta_{\text {con }}=\left\{\Delta_{\text {con }}^{+} \Delta_{\text {con }}^{-}=(1-x)^{2}-y x^{2}=0\right\}$ (with component divisors $\left.\Delta_{\text {con }}^{ \pm}=\{((1-x) \pm x \sqrt{y})\}\right)$ and the weak coupling divisor $\Delta_{\text {weak }}=\{y=0\}$ is resolved by blowing up $p$ to the exceptional divisor $E \cong \mathbf{P}^{\mathbf{1}}$ and the divisors $\Delta_{\text {con }}^{+}, \Delta_{\text {con }}^{-}, \Delta_{\text {weak }}$ meet the $u$-plane $E$ in the three special points $\tilde{u}=+1,-1, \infty$. Studying the classical field theory limit ${ }^{29}$ at $\Delta_{\text {weak }} \cap E$ one chooses the coordinates $w_{1}=\frac{x \sqrt{y}}{1-x}=1 / \tilde{u}$ and

\footnotetext{
${ }^{29}$ where classical gauge group enhancement to $S U(2)$ occurs at $a=0$; the coordinate choice reflects
} 
$w_{2}=1-x \sim \alpha^{\prime} u$ (the latter up to higher corrections in $\alpha^{\prime}$ ); at the conifold branch $\Delta_{\text {con }}^{+} \cap E$ one uses $x_{+}=\frac{1-x}{x \sqrt{y}}-1=\tilde{u}-1$ and $x_{2}=w_{2}$. One finds [24] as leading terms at $u=\infty$ and the monopole point, respectively $\left(s=2 \pi i S \propto \frac{2 \pi i}{g^{2}}, y=e^{-S} ; \kappa=\frac{i}{\pi}(\log 8-1)\right)$

\begin{tabular}{l||c|c|c}
$\Xi^{A}$ & field theory & $\Delta_{\text {weak }} \cap E$ & $\Delta_{\text {con }}^{+} \cap E$ \\
\hline$\Xi^{1}$ & 1 & 1 & 1 \\
$\Xi^{2}$ & $\alpha^{\prime} u$ & $w_{2}$ & $x_{2}$ \\
$\Xi^{3}$ & $\sqrt{\alpha^{\prime}} a$ & $\frac{i}{\pi} \sqrt{w_{2}}$ & $-\frac{\Xi^{6}}{2 \pi i} \log x_{+}+\left(1+\frac{i}{\pi}-2 \kappa\right) \Xi^{6}$ \\
$\Xi^{4}$ & $s$ & $\frac{\Xi^{1}}{\pi i} \log \left(w_{1} w_{2}\right)$ & $\frac{\Xi^{1}}{\pi i} \log x_{2}-\frac{1}{\pi i} \log \left(1-x_{+}\right)$ \\
$\Xi^{5}$ & $\alpha^{\prime} u s$ & $\frac{\Xi^{2}}{\pi i} \log \left(w_{1} w_{2}\right)$ & $\frac{\Xi^{2}}{\pi i} \log x_{2}-\frac{x_{2}}{\pi i} \log \left(1-x_{+}\right)$ \\
$\Xi^{6}$ & $\sqrt{\alpha^{\prime}} a_{D}$ & $\frac{\Xi^{3}}{\pi i} \log w_{1}+\kappa \Xi^{3}$ & $\frac{1}{\sqrt{2} \pi} \sqrt{x_{2}} x_{+}$
\end{tabular}

The periods $\Xi$ in the field theory limit are related to the periods $\Xi_{\infty}=$ $\left(1, t, s ; \partial_{s} \mathcal{F}, \partial_{t} \mathcal{F}, 2 \mathcal{F}-s \partial_{s} \mathcal{F}-t \partial_{t} \mathcal{F}\right)$ in the large complex structure basis (E.8) by ${ }^{30}$

$$
\left(\begin{array}{l}
\Xi^{1} \\
\Xi^{2} \\
\Xi^{3} \\
\Xi^{4} \\
\Xi^{5} \\
\Xi^{6}
\end{array}\right)=\left(\begin{array}{cccccc}
0 & 2 i A_{+} B & 0 & A_{-} B & 0 & 0 \\
0 & 2 i B & 0 & B & 0 & 0 \\
1 & 0 & 0 & -1 / 2 & 0 & 0 \\
0 & r_{1}+2 i A_{-} B & 2 A_{-} B & A_{-} B+i r_{2} & -i A_{+} B & -i A_{-} B \\
0 & r_{3}+2 i B & 2 B & B+i r_{4} & i B & B \\
0 & 0 & 0 & 0 & 0 & 1
\end{array}\right)\left(\begin{array}{l}
\Xi_{\infty}^{1} \\
\Xi_{\infty}^{2} \\
\Xi_{\infty}^{3} \\
\Xi_{\infty}^{4} \\
\Xi_{\infty}^{5} \\
\Xi_{\infty}^{6}
\end{array}\right)
$$

\section{F $\quad N=2$ Schwinger computation}

For four-dimensional $N=2$ supersymmetric compactification of the type IIA string on a Calabi-Yau $X$ the supermultiplet $W=F^{+} \cdots+\theta^{2} R^{+}$, containing the self-dual parts of the gravi-photon field strength and the curvature, respectively, leads in the four-dimensional effective action to terms determined by just string $g$-loop contributions [30]

$$
\int d^{4} x d^{4} \theta F_{g}\left(t_{i}\right)\left(W^{2}\right)^{g}=F_{g}\left(t_{i}\right) \int d^{4} x F_{+}^{2 g-2} R_{+}^{2}
$$

with the Kahler-moduli dependent functions $F_{g}$ (the genus $g$ topological partition function) computable from world-sheet instantons (holomorphic maps $W S \rightarrow C Y$ ) as

$$
F_{g}=\sum_{h o l o} e^{-A\left(\Sigma_{g}\right)}
$$

the double scaling limit $\epsilon \rightarrow 0$ in $y=e^{-S}=\alpha^{\prime 2} \Lambda^{4} e^{-\hat{S}}=\epsilon^{4}$ and $1-x \sim \alpha^{\prime} u=\epsilon^{2} \tilde{u}$ so that $l_{s} \Lambda=\epsilon$; the relation $m_{W_{ \pm}}^{2} \sim e^{-S / 2} \tilde{u}$ (by $u_{\text {weak }} \sim a^{2}$ and (D.11)) expresses the $\frac{8 \pi^{2}}{b_{1} g^{2}\left(M_{s t r}\right)}=-\log \frac{m_{W_{ \pm}}}{M_{s t r}}\left(b_{1}=4\right.$ )

${ }^{30}$ with $A_{ \pm}=\left(5 \pi^{4} \pm 12 \Gamma^{8}(3 / 4)\right) /\left(36 \pi^{4}\right), B=-2 \pi^{3} \sqrt{3} / \Gamma^{4}(3 / 4)$ and the $r_{i} \mathcal{O}(10)$ negative real constants 
The Kahler-moduli are scalars in the vector multiplets so the $F_{g}\left(t_{i}\right)$ are perturbatively and non-perturbatively exact as the type II dilaton lies in a hypermultiplet. Assuming the constant vev $\left\langle F^{+}\right\rangle=\lambda$ one gets as $R_{+}^{2}$ contribution $\left(F(\lambda):=\sum F_{g} \lambda^{2 g-2}\right)[30]$

$$
\left[\sum_{g} F_{g}\left(t_{i}\right) \lambda^{2 g-2}\right] R_{+}^{2}
$$

Besides the terms proportional to $\lambda^{2 g-2}$ there will be contributions to $R_{+}^{2}$ from terms like $e^{-1 / \lambda}$, leading to the full expression $\mathcal{F}_{Z}=\sum_{g} F_{g} \lambda^{2 g-2}+\mathcal{O}\left(e^{-Z / \lambda}\right)$ (with the BPS charge $Z$ of a particle in the background field made explicit). Schwinger's computation gives

$$
\mathcal{F}_{Z}=\int_{0}^{\infty} \frac{d s}{s^{3}}\left(\frac{s / 2}{\sinh s / 2}\right)^{2} e^{-Z s / \lambda}
$$

Here one may wish to think of type IIB at the conifold where a D3-brane wrapped on the vanishing $\mathbf{S}^{\mathbf{3}}$ gives rise to a massless hypermultiplet whose contribution to the $R^{2}$ term when it runs in the loop is captured by this Schwinger one-loop computation ${ }^{31}$ which integrates out the charged field to produce an effective action whose real part is a polynomial in the even powers of the field strength. With $i \mu=Z / \lambda$ one has for (F.4)

$$
\mathcal{F}_{\text {pert }}=\sum_{g} \frac{B_{g}}{2 g(2 g-2)} \mu^{2-2 g}
$$

as perturbative part ${ }^{32}$ from the large $\mu$ (small $\lambda$ ) expansion in inverse powers of the dimensionless combination $\mu=\frac{m^{2}}{2 e E}$ (in our BPS case $m=e$ ) and additional non-perturbative imaginary terms $e^{-2 \pi n \mu}$. One finds the absoprtive part (with $\tau=i \mu$, cf. (2.33))

$$
\operatorname{Im} \mathcal{F}(\mu) \sim \operatorname{Li}\left(e^{2 \pi i \tau}\right)
$$

describing the pair production of light D3D̄3 brane-antibrane states. ${ }^{33}$ [30]

${ }^{31}$ Essentially $\int_{0}^{\infty} \frac{d s}{s} \operatorname{Tr} e^{-s\left(\triangle+m^{2}\right)}=\frac{1}{4} \int_{0}^{\infty} \frac{d s}{s} \frac{1}{\sinh ^{2} \frac{s}{2}} e^{-s \frac{m^{2}}{e E}}$. To preserve at least half of the supersymmetry the background field has to be self-dual (so $\vec{E}= \pm i \vec{B}$ in Minkowski space) leading (for a boson) in the proper time formalism to the one-loop determinant expression $F(\vec{E}, \vec{B}, m)=$ $\frac{1}{2} \operatorname{Tr} \log \operatorname{det}\left((i \partial-e A)-m^{2}\right)=\frac{e^{2} E B}{2 \pi^{2}} \int_{0}^{\infty} \frac{d s}{s^{3}}\left(\frac{s / 2}{\sinh \frac{s e E}{2}}\right)\left(\frac{s / 2}{\sin \frac{s e B}{2}}\right)$ for the free energy (with $E^{2}-B^{2}=\vec{E}^{2}-\vec{B}^{2}$ and $E B=\vec{E} \cdot \vec{B}$; at the lower integration bound one has to take a UV cut-off which in string theory might be the string scale; this concerns the first two terms in (F.5) which have a divergent companion factor $\log \mu / \epsilon$ ). In the self-dual case one gets $f(\mu)=\frac{e^{2} E^{2}}{2 \pi^{2}} \int_{0}^{\infty} \frac{d s}{s^{3}} e^{-i s \mu}\left(\frac{s / 2}{\sinh \frac{s}{2}}\right)^{2}=\frac{e^{2} E^{2}}{2 \pi^{2}} \mathcal{F}(\mu)$. (F.5) gives the higher polynomial corrections to the Maxwell Lagrangian, i.e. the Euler-Heisenberg Lagrangian.

${ }^{32}$ The leading contribution at large radius in (F.2) comes from constant maps (where $\Sigma_{g}$ degenerated to a point) so the relevant moduli space replacing the sum in (F.2) is $\mathcal{M}_{g} \times X$ giving $\frac{e(X)}{2} \int_{\mathcal{M}_{g}} c_{g-1}^{3}=$ $\frac{e(X)}{2}(-1)^{g-1} e\left(\mathcal{M}_{g}\right) 2 \frac{\zeta(2 g-2)}{(2 \pi)^{2 g-2}}=\frac{e(X)}{2} \frac{B_{g}}{2 g(2 g-2)} \frac{B_{g-1}}{(2 g-2) !}$ (the Chern class referring to the Hodge bundle, given pointwise by the holomorphic one-forms, over $\mathcal{M}_{g}$ ) as leading order contribution of the $\mathcal{F}_{g}$ coefficient (cf. (F.5) for the case of the conifold).

${ }^{33}$ The $R^{2}$ term leads to $\chi$ and $\sigma$ : the action will contain terms $S=\ldots+\frac{\chi+\frac{3}{2} \sigma}{2} F\left(\lambda, t_{i}\right)+\frac{\chi-\frac{3}{2} \sigma}{2} \bar{F}\left(\bar{\lambda}, \bar{t}_{i}\right)$ so the perturbative (real) part/imaginary part corrects the Euler character/signature $\left(\lambda=g_{s}(E+i B)\right)$. 


\section{G Modular Forms and Triality symmetry}

The theta functions $\theta_{2}, \theta_{3}, \theta_{4}$ and the Eisenstein series $E_{k}$

The theta functions are defined by their series developments in $q=e^{2 \pi i \tau}$

$$
\theta_{2}(\tau)=\sum_{n \in \mathbf{Z}} q^{\frac{1}{2}\left(n+\frac{1}{2}\right)^{2}} \quad, \quad \theta_{3}(\tau)=\sum_{n \in \mathbf{Z}} q^{\frac{1}{2} n^{2}} \quad, \quad \theta_{4}(\tau)=\sum_{n \in \mathbf{Z}}(-1)^{n} q^{\frac{1}{2} n^{2}}
$$

They have the modular transformation properties

$$
\begin{array}{ll}
\theta_{2}(\tau+1)=e^{\frac{2 \pi i}{8}} \theta_{2}(\tau), & \theta_{2}(-1 / \tau)=(-i \tau)^{1 / 2} \theta_{4}(\tau) \\
\theta_{3}(\tau+1)=\theta_{4}(\tau) & , \theta_{3}(-1 / \tau)=(-i \tau)^{1 / 2} \theta_{3}(\tau) \\
\theta_{4}(\tau+1)=\theta_{3}(\tau) & , \theta_{4}(-1 / \tau)=(-i \tau)^{1 / 2} \theta_{2}(\tau)
\end{array}
$$

Because we will usually have to deal with their fourth powers we introduce the notation

$$
A:=\theta_{2}^{4} \quad, \quad-b:=B:=\theta_{3}^{4} \quad, \quad C:=\theta_{4}^{4}
$$

These fulfill the relation $A+b+C=0$ (we introduced $b$ just to keep the $\mathbf{Z}_{\mathbf{3}}$ symmetry). Let us also introduce the Eisenstein series and the absolute modular invariant $j$ (for which $j / 12^{3}$ provides an isomorphism of $\overline{S l(2, \mathbf{Z}) \backslash H}$ and $\mathbf{C}^{\infty}$, mapping $\omega, i, i \infty$ to $\left.0,1, \infty\right)$

$$
\begin{aligned}
E_{2}(\tau) & =1-24 \sum_{n=0}^{\infty} \frac{n q^{n}}{1-q^{n}}=\frac{24}{2 \pi i} \partial_{\tau} \log \eta(\tau) \\
E_{4}(\tau) & =1+240 \sum_{n=1}^{\infty} \frac{n^{3} q^{n}}{1-q^{n}}=\frac{1}{2}\left(A^{2}+b^{2}+C^{2}\right)=-3 \sigma_{2}\left(e_{i}\right) \\
E_{6}(\tau) & =1-504 \sum_{n=1}^{\infty} \frac{n^{5} q^{n}}{1-q^{n}}=-\frac{1}{2}(A-b)(b-C)(C-A)=\frac{27}{2} \sigma_{3}\left(e_{i}\right)=\frac{3}{2} \sum_{i} e_{i}^{2} \\
E_{4}^{3}-E_{6}^{2} & =12^{3} \eta^{24}=-\frac{27}{4} A b C \\
j(\tau) & =\frac{E_{4}^{3}}{\eta^{24}}=12^{3} \frac{E_{4}^{3}}{E_{4}^{3}-E_{6}^{2}}=\frac{1}{q}+744+196884 q+\cdots
\end{aligned}
$$

The half-periods $e_{i}$

An important $\mathbf{Z}_{\mathbf{3}}$ symmetric function set are the half-periods ${ }^{34}$

$$
e_{1}=\wp\left(\omega_{1} / 2\right) \quad, \quad e_{2}=\wp\left(\left(\omega_{1}+\omega_{2}\right) / 2\right) \quad, \quad e_{3}=\wp\left(\omega_{2} / 2\right)
$$

of the elliptic curve $y^{2}=4 x^{3}-g_{2} x-g_{3}=4\left(x-e_{1}\right)\left(x-e_{2}\right)\left(x-e_{3}\right)$ which are modular forms of weight two w.r.t. $\Gamma(2)$ and relate to the theta functions (with common $\sim$ factor)

$$
e_{1} \sim C-b \quad, \quad e_{2} \sim A-C \quad, \quad e_{3} \sim b-A
$$

\footnotetext{
${ }^{34}$ There are different conventions about the $e_{i}$ in the literature; one finds also $e_{2}$ and $e_{3}$ interchanged.
} 
The action of the triality group is based on the isomorphism ${ }^{35} \Sigma_{3} \cong S l(2, \mathbf{Z}) / \Gamma(2)$ (cf. (G.15), (G.16)). A,b, $C$ and the $e_{i}$ are cyclically permuted under $\mathbf{Z}_{\mathbf{3}}, \beta$ induces on the $e_{i} d \tau$ the permutation ${ }^{37} P_{\beta}$. One has to adjust the actions on the upper half-plane variable $\tau$ for the order two elements $\alpha \beta^{i}$ (initially acting as in (G.16)) involving the inversion $\alpha: \tau \rightarrow \frac{1}{\tau}$; to stay within the upper half-plane ${ }^{36}$ we define the adjusted operation

$$
\gamma_{H} \tau=\operatorname{sign}(\gamma) \cdot \gamma_{S l(2)} \tau
$$

The corresponding operations, such as $\tau \rightarrow \frac{-1}{\tau}$, are the ones which are actually induced from the $S l(2, \mathbf{Z})$ action. One has then (with (G.2) and $\left.d\left(\left(\begin{array}{ll}a & b \\ c & d\end{array}\right) \tau\right)=\frac{1}{(c \tau+d)^{2}} d \tau\right)$

$$
\begin{aligned}
& A d \tau \stackrel{\tau \rightarrow \tau+1}{\longrightarrow}-A d \tau \quad, \quad b d \tau \stackrel{\tau \rightarrow \tau+1}{\longrightarrow}-C d \tau \quad, \quad C d \tau \stackrel{\tau \rightarrow \tau+1}{\longrightarrow}-b d \tau \\
& A d \tau \stackrel{\alpha_{H}}{\longrightarrow}-C d \tau \quad, \quad b d \tau \stackrel{\alpha_{H}}{\longrightarrow}-b d \tau \quad, \quad C d \tau \stackrel{\alpha_{H}}{\longrightarrow}-A d \tau
\end{aligned}
$$

and so $\alpha_{H}$ induces the permutation $P_{\alpha_{H}}=\left(\begin{array}{ccc}1 & 2 & 3 \\ 3 & 2 & 1\end{array}\right)$, i.e. $\left.e_{1} d \tau\right|_{\alpha_{H} \tau}=e_{3} d \tau,\left.e_{2} d \tau\right|_{\alpha_{H} \tau}=$ $e_{2} d \tau,\left.e_{3} d \tau\right|_{\alpha_{H} \tau}=e_{1} d \tau$. So $\Sigma_{3}$ operates on $e_{i} d \tau$ via the permutation $\operatorname{action}^{37}$

$$
\left.e_{i} d \tau\right|_{\gamma_{H} \tau}=e_{P_{\gamma}(i)} d \tau
$$

\section{The Legendre $\lambda$ function}

The Legendre function ${ }^{38}$ is defined as a cross ratio (cf. below) of the half-periods

$$
\lambda(\tau)=\frac{e_{2}-e_{3}}{e_{1}-e_{3}}=-\frac{A}{b}
$$

which is a modular function for $\Gamma(2)$ and transforms under $\Sigma_{3}=S l(2, \mathbf{Z}) / \Gamma(2)$ as follows

$$
\begin{gathered}
\lambda(\tau)=\lambda(\tau) \quad, \quad \lambda\left(\frac{1}{1-\tau}\right)=\frac{1}{1-\lambda(\tau)} \quad, \quad \lambda\left(\frac{\tau-1}{\tau}\right)=\frac{\lambda(\tau)-1}{\lambda(\tau)} \\
\lambda\left(-\frac{1}{\tau}\right)=1-\lambda(\tau) \quad, \quad \lambda(\tau-1)=\frac{\lambda(\tau)}{\lambda(\tau)-1} \quad, \quad \lambda\left(\frac{\tau}{1-\tau}\right)=\frac{1}{\lambda(\tau)}
\end{gathered}
$$

i.e. $\quad \lambda(\gamma \tau)=\gamma \lambda(\tau)$ for $\gamma \in \mathbf{Z}_{\mathbf{3}}$ and $\lambda\left(\gamma_{H} \tau\right)=\gamma \beta \lambda(\tau)$ for $\gamma \in \Sigma_{3} \backslash \mathbf{Z}_{\mathbf{3}}$. For the $\mathbf{Z}_{\mathbf{3}}$ transforms of $\lambda$ (generalising (G.11)) and the absolute modular invariant function $j(\tau)$ ( $\lambda$ provides an isomorphism of $\overline{\Gamma(2) \backslash H}$ and $\mathbf{C}^{\infty}$, mapping $i \infty, 0,1$ to $\left.0,1, \infty\right)$ one has

$$
\begin{aligned}
\lambda & =-\frac{A}{b}=\frac{e_{2}-e_{3}}{e_{1}-e_{3}}, \beta \lambda=-\frac{b}{C}=\frac{e_{3}-e_{1}}{e_{2}-e_{1}}, \beta^{2} \lambda=-\frac{C}{A}=\frac{e_{1}-e_{2}}{e_{3}-e_{2}}(\mathrm{G} \\
\frac{3^{3}}{2^{2}} j & =(\lambda-\beta \lambda) \cdot\left(\beta \lambda-\beta^{2} \lambda\right) \cdot\left(\beta^{2} \lambda-\lambda\right)=\frac{(\lambda+\omega)^{3}\left(\lambda+\omega^{2}\right)^{3}}{\lambda^{2}(1-\lambda)^{2}}
\end{aligned}
$$

\footnotetext{
$35\left(\begin{array}{ll}1 & 0 \\ 0 & 1\end{array}\right),\left(\begin{array}{cc}0 & 1 \\ -1 & 1\end{array}\right),\left(\begin{array}{cc}1 & -1 \\ 1 & 0\end{array}\right)$ and $\left(\begin{array}{cc}0 & -1 \\ 1 & 0\end{array}\right),\left(\begin{array}{cc}1 & -1 \\ 0 & 1\end{array}\right),\left(\begin{array}{cc}1 & 0 \\ -1 & 1\end{array}\right)$ give representatives

$36\left(\begin{array}{ll}0 & 1 \\ 1 & 0\end{array}\right) \notin S l(2, \mathbf{Z})$, and $\operatorname{Im}\left(\left(\begin{array}{ll}a & b \\ c & d\end{array}\right) \tau\right)=\frac{1}{|c \tau+d|^{2}} \operatorname{Im} \tau$ gets an additional determinantal factor

$37 P_{\beta}=\left(\begin{array}{lll}1 & 2 & 3 \\ 2 & 3 & 1\end{array}\right), P_{\alpha_{H}}=\left(\begin{array}{ccc}1 & 2 & 3 \\ 3 & 2 & 1\end{array}\right), P_{(\alpha \beta)_{H}}=\left(\begin{array}{ccc}1 & 2 & 3 \\ 1 & 3 & 2\end{array}\right), P_{\left(\alpha \beta^{2}\right)_{H}}=\left(\begin{array}{ccc}1 & 2 & 3 \\ 2 & 1 & 3\end{array}\right)$

${ }^{38}$ Note that here again conventions can differ, especially from a different choice of the $e_{i}$. By (G.15) the relations (G.13) can also be understood as permutations in (G.11) for the case $\infty, e_{1}, e_{2}, e_{3}$
} 
The cross ratio $z=\mathrm{cr}_{z_{1}, z_{2}, z_{3}, z_{4}}=\frac{z_{1}-z_{3}}{z_{1}-z_{4}} / \frac{z_{2}-z_{3}}{z_{2}-z_{4}}$ of four points $z_{1}, z_{2}, z_{3}, z_{4}$ of $\mathbf{P}^{\mathbf{1}}(\mathbf{C})$ gives, as one has the equalities $c r_{z_{1}, z_{2}, z_{3}, z_{4}}=c r_{z_{2}, z_{1}, z_{4}, z_{3}}=c r_{z_{3}, z_{4}, z_{1}, z_{2}}=c r_{z_{4}, z_{3}, z_{2}, z_{1}}$ but the index four subgroup $\Sigma_{3}$ operates effectively, a realisation of the isomorphism $\operatorname{Sl}(2, \mathbf{Z}) / \Gamma(2) \cong \Sigma_{3}$

$$
1 \longrightarrow V \rightarrow \Sigma_{4} \longrightarrow S l(2, \mathbf{Z}) / \Gamma(2) \cong \Sigma_{3} \longrightarrow 1
$$

considering a non-linear action of the triality symmetry group $\Sigma_{3}$ on $z$

$$
\begin{array}{ccrlrl}
z & \beta z & =\frac{1}{1-z} & \beta^{2} z & =\frac{z-1}{z} \\
\alpha z=\frac{1}{z} & \alpha \beta z & =1-z & \alpha \beta^{2} z & =\frac{z}{z-1}
\end{array}
$$

The hypergeometric function $F_{\frac{1}{2} \frac{1}{2} 1}$ and the Schwarzian

Gauss's hypergeometric function ${ }_{2} F_{1}(\alpha, \beta, \gamma ; z)=: F_{\alpha \beta \gamma}(z)$ solves the differential equation

$$
z(1-z) \frac{d^{2} F}{d z^{2}}+(\gamma-(\alpha+\beta+1) z) \frac{d F}{d z}-\alpha \beta F=0
$$

is given for $|z|<1$ by $F_{\alpha \beta \gamma}(z)=\frac{\Gamma(\gamma)}{\Gamma(\alpha) \Gamma(\beta)} \sum_{n=0}^{\infty} \frac{\Gamma(\alpha+n) \Gamma(\beta+n)}{\Gamma(\gamma+n)} \frac{z^{n}}{n !}$ and has the Euler integral

$$
F_{\alpha \beta \gamma}(z)=\frac{\Gamma(\gamma)}{\Gamma(\beta) \Gamma(\gamma-\beta)} \int_{0}^{1} t^{\beta-1}(1-t)^{\gamma-\beta-1}(1-z t)^{-\alpha} d t
$$

We are interested mainly in $F(z):=F_{\frac{1}{2} \frac{1}{2} 1}(z)$ which gives the inverse function to $\lambda$ by

$$
\tau(\lambda)=i \frac{F(1-\lambda)}{F(\lambda)}
$$

The $\Sigma_{3}$ covariance properties (G.12) of $\lambda$ are equivalent to corresponding properties of $F$

$$
F\left(\alpha \beta^{2} z\right)=\sqrt{\alpha \beta z} F(z) \quad, \quad F(\alpha z)=\sqrt{z}(F(z)-i F(\alpha \beta z))
$$

For a function $z(x)$ one defines the Schwarzian derivative

$$
\{z, x\}=\frac{z^{\prime \prime \prime}}{z^{\prime}}-\frac{3}{2}\left(\frac{z^{\prime \prime}}{z^{\prime}}\right)^{2}
$$

which is $S l(2, \mathbf{C})$ invariant $\left\{\frac{a z+b}{c z+d}, x\right\}=\{z, x\}$ (so a fractional linear $z(x)$ solves $\{z, x\}=0$ ) and has the composition rule $\{y, x\}=\{y, z\}\left(\frac{d z}{d x}\right)^{2}+\{z, x\}$ (implying the inversion $\left.\{x, z\}=-\left(\frac{d x}{d z}\right)^{2}\{z, x\}\right)$. For two independent solutions $y_{1}, y_{2}$ of a second order differential equation the quotient $z(x)=y_{2}(x) / y_{1}(x)$ satisfies a Schwarzian differential equation

$$
\frac{d^{2} y}{d x^{2}}+p(x) \frac{d y}{d x}+q(x) y=0 \quad \Longrightarrow \quad\{z, x\}=-\frac{p^{2}}{2}-p^{\prime}+2 q
$$


The dilogarithm

$L i=L i_{2}=\sum_{n \geq 1} x^{n} / n^{2}$ (cf. (2.1) and [8]) is member of a series of higher polylogarithms $\left(L i_{1}(x)=\sum_{n \geq 1} x^{n} / n=\log \beta x \quad, L i_{0}(x)=\sum_{n \geq 1} x^{n}=x \cdot \beta x\right)$ with a hierarchical relation

$$
\frac{d}{d x} L i_{k+1}\left(e^{x}\right)=L i_{k}\left(e^{x}\right)
$$

We describe $L i$ on the critical circle $|u|=1$, the boundary of the domain of convergence of the series representation. One has the elementary evaluation for the real part

$$
\operatorname{Re} \operatorname{Li}\left(e^{i \phi}\right)=\sum_{n \geq 1} \frac{\cos n \phi}{n^{2}}=\frac{\pi^{2}}{6}-\frac{1}{4} \phi(2 \pi-\phi)
$$

and the non-elementary odd function (of period $2 \pi$ ) $I(\phi)$ for the imaginary part ${ }^{39}$

$$
I(\phi):=\operatorname{Im} L i\left(e^{i \phi}\right)=\sum_{n \geq 1} \frac{\sin n \phi}{n^{2}}=-\int_{0}^{\phi} \log \left(2 \sin \frac{\psi}{2}\right) d \psi
$$

Let us give the monodromies for the dilogarithm. Its differential equation ${ }^{40}$ is

$$
\frac{d L i}{d \log u}=\log \beta u
$$

The monodromy representation of the relevant fundamental group $\pi_{1}\left(\mathbf{P}^{\mathbf{1}} \backslash\{0,1, \infty\}\right.$ ) (of loops based at $1 / 2$, say) describes the images of the generator loops $l_{i}(t)(i=0,1$, $t \in[0,1]$ ) which encircle (in the mathematically positively oriented sense) $z=0$ and $z=1$, respectively (then $l_{\infty} \circ l_{1} \circ l_{0}=1$ ). One has as multi-valuedness

$$
\begin{aligned}
\log z & \stackrel{l_{0}}{\longrightarrow} \log z+2 \pi i \\
\log \beta z & \stackrel{l_{1}}{\longrightarrow} \log \beta z-2 \pi i \quad, \quad \operatorname{Li}(z) \stackrel{l_{1}}{\longrightarrow} \operatorname{Li}(z)-2 \pi i \log z
\end{aligned}
$$

Now take as fundamental object a principal branch (on $|z-1 / 2|<1 / 2$ ) of

$$
L(z)=\left(\begin{array}{ccc}
1 & \log \beta z & \operatorname{Li}(z) \\
0 & 1 & \log z \\
0 & 0 & 1
\end{array}\right)
$$

Analytic continuation along a loop $l$ in $\mathbf{P}^{\mathbf{1}} \backslash\{0,1, \infty\}$ leads to $M(l) L(z)$ where

$$
M: \pi_{1}\left(\mathbf{P}^{\mathbf{1}} \backslash\{0,1, \infty\}\right) \rightarrow G l(3, \mathbf{C})
$$

defines the monodromy representation. One finds the monodromy matrices (2.2). There are two equivalent ways to describe this: in the vector resp. Heisenberg picture one has the matrices $M\left(l_{i}\right)$ of (2.2) resp. the elements (2.4) expressing the monodromies (G.27) for the vector $c_{3}$ of (2.2) resp. the group element (2.3).

\footnotetext{
${ }^{39}$ in general one has to take the absolute value of the expression the log of which occurs here

${ }^{40}$ An additional constant $\log \beta u_{*}$ in $\frac{d T}{d \log u}=\log \beta u-\log \beta u_{*}$ would give $T(u)=L i(u)-\log \beta u_{*} \log u$.
} 


\section{References}

1. M. Atiyah and E. Witten, "M-Theory Dynamics On A Manifold Of $G_{2}$ Holonomy", hep-th/0107177.

2. H. Ooguri and C. Vafa, "Summing up D-Instantons", Phys. Rev. Lett. 77 (1996) 3296, hep-th/9608079.

3. N. Seiberg and S. Shenker, "Hypermultiplet Moduli Space and String Compactification to Three Dimensions", Phys. Lett. B388 (1996) 521, hep-th/9608086.

4. T.R. Taylor and C. Vafa, "RR Flux on Calabi-Yau and Partial Supersymmetry Breaking”, Phys. Lett. B474 (2000) 130, hep-th/9912152.

5. F. Cachazo, K. Intriligator and C. Vafa, "A Large $N$ Duality via a Geometric Transition", Nucl. Phys. B 603 (2001) 3, hep-th/0103067.

6. M. Atiyah, J. Maldacena and C. Vafa, "An M-theory Flop as a Large N Duality", hep-th/0011256.

7. M. Aganagic and C. Vafa, "Mirror Symmetry and a $G_{2}$ Flop", hep-th/0105225.

8. G. Curio, Superpotentials for $M$-theory on a $G_{2}$ holonomy manifold and triality symmetry, hep-th/0212211.

9. D. Joyce, "On counting special Lagrangian homology 3-spheres", hep-th/9907013.

10. A. Brandhuber, J. Gomis, S.S. Gubser and S. Gukov, "Gauge Theory at Large N and New $G_{2}$ Holonomy Metrics", Nucl.Phys. B611 (2001) 179, hep-th/0106034.

11. J. Schwinger, "On Gauge Invariance and Vacuum Polarization", Phys. Rev. 82 (1951) 664.

V. Weisskopf, Kgl. Danske Videnskab. Selskabs. Met.-fys. Medd. 14 No. 6 (1936). K. Srinivasan and T. Padmanabhan, "Facets of Tunneling: Particle production in external fields", gr-qc/9807064; "Particle production and complex path analysis", Phys. Rev. D60 (1999) 024007, gr-qc/9812028.

G.V. Dunne and Th.M. Hall, "Borel Summation of the Derivative Expansion and Effective Actions", Phys. Rev. D60 (1999) 065002, hep-th/9902064; "On the QED Effective Action in Time Dependent Electric Backgrounds", Phys. Rev. D58 (1998) 105022, hep-th/9807031. 
A.B. Balantekin, J.E. Seger and S.H. Fricke, "Dynamical Effects in Pair Production by Electric Fields", Int. Jour. Mod. Phys. A6 No. 5 (1991) 695.

V.B. Adesi and S. Zerbini, "Analytic continuation of the Hurwitz Zeta Function with physical application", to be published in J. Math. Phys, hep-th/0109136.

V.P. Gusynin and I.A. Shovkovy, "Derivative Expansion of the Effective Action for QED in 2+1 and 3+1 dimensions", J. Math. Phys. 40 (1999) 5406, hepth/9804143.

R. Soldati and L. Sorbo, "Effective action for Dirac spinors in the presence of general uniform electromagnetic fields", Phys. Lett. B426 (1998) 82, hep-th/9802167. I.N. McArthur and T.D. Gargett, "A "Gaussian" Approach to Computing Supersymmetric Effective Actions", Nucl. Phys. B497 (1997) 525, hep-th/9705200.

S.K. Blau, M. Visser and A. Wipf, "Analytic Results for the Effective Action", Int. Jour. Mod. Phys A6 No. 30 (1991) 5409.

12. S.P. Kim and D.N. Page, "Schwinger Pair Production via Instantons in Strong Electric Fields", to appear in Phys. Rev. D, hep-th/0005078.

13. M.B. Green and M. Gutperle, "D-instanton partition functions". Phys.Rev. D58 (1998) 046007, hep-th/9804123.

14. K. Becker and M. Becker, "Instanton Action for Type II Hypermultiplets", Nucl. Phys. B551 (1999) 102, hep-th/9901126.

15. M. Gutperle and M. Spalinski, "Supergravity Instantons and the Universal Hypermultiplet”, JHEP 0006 (2000) 037, hep-th/0005068 .

16. O.J. Ganor, "U-duality Twists and Possible Phase Transitions in 2+1D Supergravity” Nucl. Phys. B549 (1999) 145, hep-th/9812024.

17. S.V. Ketov, "Universal hypermultiplet metrics", Nucl. Phys. B604 (2001) 256, hep-th/0102099; "D-instantons and universal hypermultiplet", hep-th/0112012.

18. S. Katz, A. Klemm and C. Vafa, "Geometric Engineering of Quantum Field Theories", Nucl. Phys. B497 (1997) 173, hep-th/9609239.

19. N. Seiberg and E. Witten, "Monopole Condensation, And Confinement In N=2 Supersymmetric Yang-Mills Theory", Nucl. Phys. B426 (1994) 19, Erratum-ibid. B430 (1994) 485, hep-th/9407087. 
20. N. Seiberg and E. Witten, "Monopoles, Duality and Chiral Symmetry Breaking in N=2 Supersymmetric QCD”, Nucl. Phys. B431 (1994) 484, hep-th/9408099.

21. M. Matone, "Instantons and recursion relations in N=2 Susy gauge theory", Phys. Lett. B357 (1995) 342, hep-th/9506102.

22. A. Klemm, "On the Geometry behind $N=2$ Supersymmetric Effective Actions in Four Dimensions", hep-th/9705131.

23. S. Kachru, A. Klemm, W. Lerche, P. Mayr and C. Vafa, "Nonperturbative Results on the Point Particle Limit of N=2 Heterotic String Compactifications", Nucl. Phys. B459 (1996) 537, hep-th/9508155.

24. G. Curio, A. Klemm, D. Lüst and S. Theisen, "On the Vacuum Structure of Type II String Compactifications on Calabi-Yau Spaces with H-Fluxes", Nucl. Phys. B609 (2001) 3, hep-th/0012213.

25. G. Curio, B. Kors and D. Lüst, "Fluxes and Branes in Type II Vacua and M-theory Geometry with G(2) and Spin(7) Holonomy", hep-th/0111165.

26. N. Nekrasov, "Five Dimensional Gauge Theories and Relativistic Integrable Systems", Nucl. Phys. B531 (1998) 323, hep-th/9609219.

27. A. Lawrence and N. Nekrasov, "Instanton sums and five-dimensional gauge theories", Nucl. Phys. B513 (1998) 239, hep-th/9706025.

28. H. Kanno and Y. Ohta, "Picard-Fuchs Equation and Prepotential of Five Dimensional SUSY Gauge Theory Compactified on a Circle”, Nucl. Phys. B530 (1998) 73 , hep-th/9801036.

29. T. Eguchi and H. Kanno, "Five-Dimensional Gauge Theories and Local Mirror Symmetry" Nucl. Phys. B586 (2000) 331, hep-th/0005008.

30. R. Gopakumar and C. Vafa, "M-Theory and Topological Strings-I, II", hepth/9809187, 9812127. "Topological Gravity as Large N Topological Gauge Theory", hep-th/9802016, Adv. Theor. Math. Phys. 2 (1998) 413. "On the Gauge Theory/Geometry Correspondence", hep-th/9811131, Adv. Theor. Math. Phys. 3 (1999) 1415.

31. C. Vafa, "Superstrings and Topological Strings at Large N", J.Math.Phys. 42 (2001) 2798 , hep-th/0008142. 